\title{
Fluid-fluid phase separation in colloid-polymer mixtures studied with small angle light scattering and light microscopy
}

\author{
Nynke A.M. Verhaegh, Jeroen S. van Duijneveldt ${ }^{1}$, Jan K.G. Dhont, \\ Henk N.W. Lekkerkerker* \\ Van't Hoff Laboratory, Utrecht University, Padualaan 8, 3584 CH Utrecht, The Netherlands
}

Received 2 February 1996; revised 4 April 1996

\begin{abstract}
Mixtures of colloidal silica spheres and polydimethylsiloxane in cyclohexane with a colloid-polymer size ratio of about one were found to phase separate into two fluid phases, one which is colloid-rich and one which is colloid-poor. In this work the phase separation kinetics of this fluid-fluid phase separation is studied for different compositions of the colloid-polymer mixtures, and at several degrees of supersaturation, with small angle light scattering and with light microscopy. The small angle light scattering curve exhibits a peak that grows in intensity and that shifts to smaller wave vector with time. The characteristic length scale that is obtained from the scattering peak is of the order of a few $\mu \mathrm{m}$, in agreement with observations by light microscopy. The domain size increases with time as $t^{1 / 3}$, which might be an indication of coarsening by diffusion and coalescence, like in the case of binary liquid mixtures and polymer blends. For sufficiently low degrees of supersaturation the angular scattering intensity curves satisfy dynamical scaling behavior.
\end{abstract}

\section{Introduction}

Mixtures of colloidal hard spheres and non-adsorbing polymer show interesting phase behavior, as several experimental studies have revealed [1-13]. The starting point for theoretical models describing this phase behavior is the notion that the polymers are excluded from a depletion zone surrounding the particles, since they cannot penetrate the colloidal spheres $[14,15]$. The free volume available to polymers is therefore limited to the total volume minus the volume occupied by the colloidal spheres and their depletion zones. On approach of the colloidal particles these depletion zones overlap, yielding an increase in free volume available to the polymers.

\footnotetext{
* Corresponding author.

${ }^{1}$ Present address: Department of Physics, University of Bristol. Tyndall Avenue, Bristol, BS8 1TL. England.
} 
This is equivalent to an effective attraction between the colloidal particles (depletion attraction) which in turn gives rise to clustering.

The range and depth of this depletion attraction can be tuned by the size and concentration of the polymer. Due to this attraction, phase separation can occur into a colloid-rich phase and a colloid-poor phase. A thermodynamic perturbation theory, in which the interaction potential consists of a hard sphere reference potential and a depletion attraction as a perturbation, has been used to predict phase diagrams [16-18]. The co-existing phases can be described as a colloidal gas, a colloidal liquid and a colloidal solid. Effectively, the ratio between the polymer radius $r_{\mathrm{p}}$ and colloid radius $r_{\mathrm{c}}$ determines what kind of phases are involved: in case $r_{\mathrm{p}} / r_{\mathrm{c}}<0.3$ the phase diagram exhibits a co-existence region between a colloidal crystal and a colloidal fluid. In case $r_{\mathrm{p}} / r_{\mathrm{c}}>0.3$ a gas-liquid co-existence also appears in the phase diagram. If polymer partitioning is taken into account [18] a three-phase equilibrium region in the latter phase diagram is found. These predicted phase diagrams have recently been confirmed experimentally including the existence of a three-phase gas-liquid-crystal region $[1-3]$.

The kinetics of these phase separations in colloid-polymer mixtures is also a matter of current interest. The kinetics of solid-fluid phase separation in mixtures with narrow depletion attractions have been studied experimentally [4,5]. These studies have revealed that in addition to the well-known nucleation and growth and spinodal decomposition scenarios aggregation and gelation also play a role.

In the present work we study the phase separation kinetics in mixtures of small silica (Ludox) spheres and polydimethylsiloxane of size ratio of about one, under conditions where they display fluid-fluid phase separation. As far as we know this is the first time that fluid-fluid phase separation kinetics have been studied in mixtures of colloids and non-adsorbing polymers. In principle this system should also exhibit a solid phase at sufficiently high polymer concentrations but we did not investigate this. In any case, the high polydispersity of the silica spheres $(19 \%)$ will prohibit the occurrence of a crystalline phase [19-21].

Because of the small difference in refractive index between the particles and solvent, leading to sufficiently transparent systems, and because of the appropriate length $[\mu \mathrm{m}]$ and time scales [s] during the unmixing process, the phase separation kinetics could be conveniently studied with conventional light microscopy and with small angle light scattering (SALS). Because of the small size of the particles the dynamics of phase separation is fast compared to the time scale on which sedimentation of the colloidal spheres or polymers has to be taken into account. Light microscopy yielded real-space information about the time-dependent structure evolution during phase separation. Variations in composition gave rise to refractive-index fluctuations which lead to images. Domains with different intensities were observed, which coarsened in time, and could be followed during several minutes. The small angle light scattering of a phase separating system showed a ring in the scattering pattern with its maximum at wave vector $q_{\max }$, indicative of a characteristic length scale in the system equal to 
$2 \pi / q_{\max }$. The intensity and location of this ring changed in time yielding information about the structure development of the system during the first minutes.

In order to analyse the phase separation kinetics in our colloid-polymer mixtures we compared our results with theories developed for liquid-liquid phase separation, that have already been applied in a wide range of dissimilar systems such as binary liquid mixtures [22, 23], metallic alloys and inorganic glasses [24, 25], polymer blends and mixtures [26,27], micelles [28] and thermodynamically unstable colloidal systems $[29,30]$. Following Cahn $[25,31-34]$ it has become customary to distinguish between two kinds of mechanism which describe the early stage dynamics of phase separation: spinodal decomposition in thermodynamically unstable systems versus nucleation and growth in thermodynamically metastable systems. For spinodal decomposition (unstable) delocalized, long range density variations develop in time leading to a bicontinuous network. For nucleation a localized nucleus (large amplitude density fluctuation) of a metastable new phase is formed. The nucleus coarsens in time, if it is above a critical size, yielding a morphology of isolated droplets.

Binder [35-37] has emphasized that the transition from nucleation to spinodal decomposition is gradual, and even then the distinction can be made only for the initial stage of phase separation. In later stages phase separation proceeds as the evolution of a structure consisting of domains of different compositions corresponding to the co-existing phases (in our case colloid-rich and colloid-poor). The structure coarsening with time during unmixing is usually found to satisfy a power law behavior. Initially coarsening is due to either a diffusion-reaction process describing the coalescence of diffusing domains [38], or an evaporation-condensation process describing the evaporation of particles from small domains and condensation into large domains $[39,40]$. At later times the coarsening is dominated by hydrodynamic flow [41], until the domains grow larger than the capillary length, when coarsening is influenced by gravity [41]. Finally, sedimentation of denser domains and creaming of less dense domains yield a macroscopically phase-separated system.

Binder [35, 38] and Marro and Lebowitz $[42,43]$ have proposed a dynamical scaling relation for the structure factor of unmixing processes in binary mixtures and metallic alloys. They assumed that the phase separation can be described with one time-dependent parameter, which should be the domain size.

We analyzed the structure evolution in our unmixing colloid-polymer mixtures in terms of the theories mentioned above. Specifically, the time dependence of $q_{\max }$ and dynamic scaling behavior of our data have been examined. The outline of this paper is as follows. We first describe our colloid-polymer mixtures and the experiments (Section 2). Then we present the phase separation results as observed visually, and with small angle light scattering and light microscopy (Section 3). In Section 4.1 we present the experimental phase diagram. In Section 4.2 we analyze our SALS-data, first by presenting a so-called Cahn-plot (Section 4.2.1), then by discussing the coarsening behavior (Section 4.2.2) and finally by checking the dynamic scaling behavior of our scattering data. In Section 4.3 we analyze the obtained micrographs. Section 5 contains the conclusions. 


\section{Experimental}

\subsection{Sample preparation}

The spheres have been made by coating commercially available ludox spheres (Ludox AS 40\%, Dupont) with stearyl alcohol (1-octadecanol, Merck, zur synthese) in order to provide a steric stabilization [44]. They formed stable dispersions in cyclohexane (Janssen). The spheres were coded STLU (stearyl coated ludox spheres) and they were characterized with transmission electron microscopy and with dynamic light scattering (Table 1). Polydimethylsiloxane (PDMS, Janssen) has been used as received and was dispersed in cyclohexane. The radius of gyration and the molecular weight have been determined with static light scattering (Zimm plots) and with gel permeation chromatography using a multi-angle laser light scattering detector (Table 1).

Samples were contained in rectangular glass cells of $2 \mathrm{~mm}$ optical path length (Hellma). From the mass of the cuvette, the colloid volume fraction $\phi_{c}$ and the polymer volume fraction $\phi_{\mathrm{p}}$ were determined. The samples were shaken vigorously in order to homogenize the mixture. Even though it was not clear whether the sample was homogeneous on a particle level, it was checked that the measurements were reproducible if performed immediately after each other. Time zero was defined to be the moment at which the shaking was stopped. The first measurement could then be taken a few seconds later.

\subsection{Phase diagram}

A series of mixtures was prepared with several volume fraction ratios $\left(\phi_{\mathrm{p}} / \phi_{\mathrm{c}}\right)$. By either concentrating or diluting the samples with cyclohexane, the position of the phase boundary could be determined by visual observation. When the mixture was not stable (supersaturated) the sample became turbid and within approximately $1 \mathrm{~h}$ a sharp interface could be observed between two transparent phases. The resulting phase boundary is presented in Fig. 1. Here the volume fraction polymer coils $\phi_{\mathrm{p}}$ is

Table 1

Sample properties: $\boldsymbol{r}_{\text {TEM }}$ is the radius obtained with transmission electron microscopy, $r_{\mathrm{h}}$ the hydrodynamic radius obtained with dynamic light scattering using the method of cumulants [45], $\delta$ the polydispersity $\left(\sigma_{\mathrm{r}} /\langle\boldsymbol{r}\rangle\right), M_{\mathrm{w}}$ the molecular weight of PDMS determined with static light scattering (SLS) and with gel permeation chromatography using a multi-angle laser light scattering detector (GPC/MALLS), $r_{\mathrm{SLS}}$ and $r_{\text {GPC }}$ are the radius of gyration of PDMS as determined with respectively SLS and GPC/MALLS, and $\rho$ is the density of the particles

\begin{tabular}{|c|c|c|c|c|c|c|c|c|}
\hline \multicolumn{4}{|l|}{ STLU } & \multicolumn{5}{|c|}{ PDMS } \\
\hline $\begin{array}{l}r_{\text {TEM }} \\
{[\mathrm{nm}]}\end{array}$ & $\begin{array}{l}r_{\mathrm{h}} \\
{[\mathrm{nm}]}\end{array}$ & $\begin{array}{l}\delta \\
\%\end{array}$ & $\begin{array}{l}\rho \\
{\left[\mathrm{g} \mathrm{ml}^{-1}\right]}\end{array}$ & $M_{\mathrm{w}}$ & $M_{\mathrm{w}} / M_{\mathrm{n}}$ & $\begin{array}{l}r_{\text {sLs }} \\
{[\mathrm{nm}]}\end{array}$ & $\begin{array}{l}r_{\mathrm{GPC}} \\
{[\mathrm{nm}]}\end{array}$ & $\begin{array}{l}\rho \\
{\left[\mathrm{g} \mathrm{ml}^{-1}\right]}\end{array}$ \\
\hline 8 & 12 & 19 & 1.60 & 97000 & 1.9 & 13 & 14 & 0.976 \\
\hline
\end{tabular}




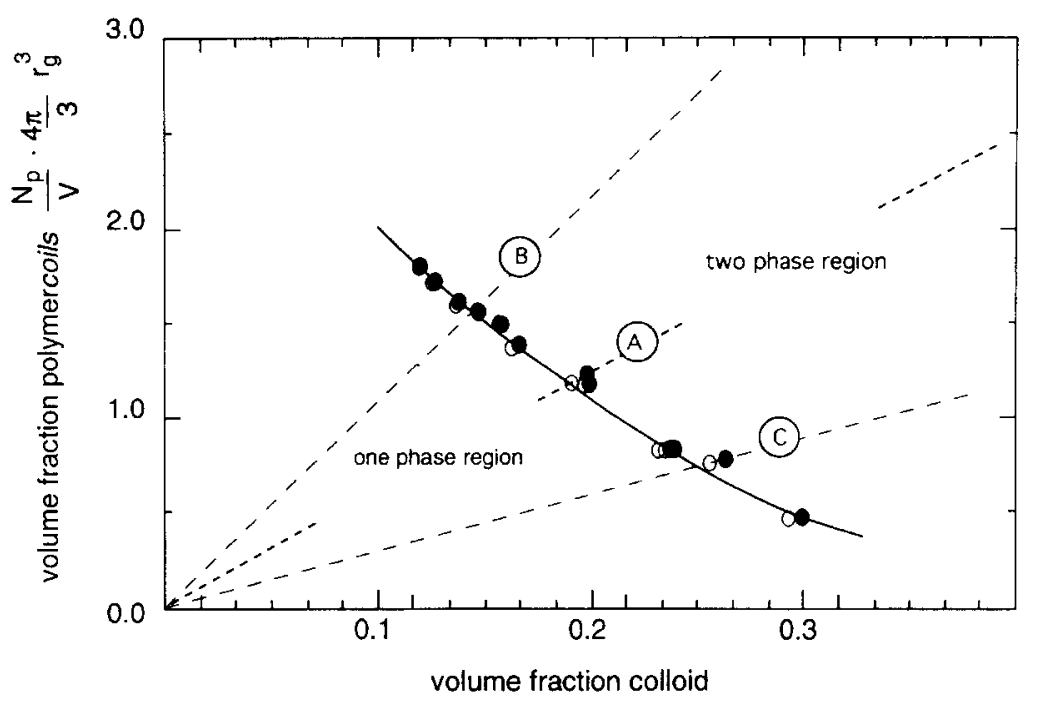

Fig. 1. Experimental phase diagram for mixtures of STLU $(r=13 \mathrm{~nm})$ and PDMS $(r=14 \mathrm{~nm})$. The filled circles represent compositions which give phase separation, and the open circles represent compositions which do not give phase separation. The lines denoted by A, B and C correspond to samples A, B and C, with volume fraction ratio $\phi_{\mathrm{p}} / \phi_{\mathrm{c}}$ respectively 6,11 and 3 . From the ratio between the volumes of colloid-rich and colloid-poor phase after phase separation we consider sample A to be almost critical.

defined as the polymer number density times a spherical volume with radius equal to the radius of gyration of the polymer.

In the following we study three volume fraction ratios indicated by line $\mathrm{A}$, line $\mathrm{B}$ and line C in Fig. 1, corresponding to $\phi_{\mathrm{p}} / \phi_{\mathrm{c}}=6.2,10.8$ and 3.0 respectively. Based upon the observation that the partial volumes of the two co-existing phases strongly depended on the volume fraction ratio, we considered sample A to be close to critical, since the partial volumes of the colloid-rich and the colloid-poor phases were almost equal. Sample B developed a larger colloid-poor phase and sample C developed a larger colloid-rich phase. Table 2 presents the overall volume fractions colloid and polymer $\left(\phi_{\mathrm{c}}^{\#}\right.$ and $\phi_{\mathrm{p}}^{*}$ ) coinciding with the phase boundary for sample A, B and C. We also included the volume fractions $\phi_{c}$ and $\phi_{p}$ of the actual experiments $A_{1}, A_{2}, A_{3}, B_{1}$, $B_{2}, C_{1}$ and $C_{2}$, for which an increase in subscript indicates a higher supersaturation.

\subsection{Small angle light scattering}

A small angle light scattering (SALS) setup was constructed, virtually identical to the apparatus described by Schätzel and Ackerson [46]. The apparatus is shown schematically in Fig. 2. It consists of a $5 \mathrm{~mW}$ randomly polarized helium-neon laser at a wavelength of $\lambda=632.8 \mathrm{~nm}$. The beam is spatially filtered and expanded using a $5 \times$ microscope objective, a $25 \mu \mathrm{m}$ pinhole and a $80 \mathrm{~mm}$ focal length output doublet (Oriel, Stratford, CT), and, passing through the sample, it is focussed onto a distant 
Table 2

Characteristics of experiments. $t_{10}$ is the time at which transmission becomes smaller than $10 \%$; $t_{\mathrm{g}}$ is the time after which the light scattering pattern is transformed due to gravitational settling

\begin{tabular}{|c|c|c|c|c|c|c|}
\hline \multirow{2}{*}{$\begin{array}{l}\text { Experiment } \\
\text { code }\end{array}$} & \multicolumn{2}{|c|}{ Phase boundary } & \multirow[t]{2}{*}{$\Phi_{\mathrm{c}}$} & \multirow[t]{2}{*}{$\Phi_{\mathrm{p}}$} & \multirow{2}{*}{$\begin{array}{l}t_{10} \\
{[s]}\end{array}$} & \multirow{2}{*}{$\begin{array}{l}t_{\mathrm{g}} \\
{[\mathrm{s}]}\end{array}$} \\
\hline & $\Phi_{\mathrm{c}}^{*}$ & $\Phi_{\mathrm{p}}^{\#}$ & & & & \\
\hline A & 0.195 & 1.21 & & & & \\
\hline $\mathrm{A} 1$ & & & 0.201 & 1.25 & 87 & 40 \\
\hline A2 & & & 0.202 & 1.26 & 30 & 20 \\
\hline A 3 & & & 0.205 & 1.28 & $<1$ & $>13$ \\
\hline B & 0.146 & 1.58 & & & & \\
\hline B1 & & & 0.147 & 1.58 & $>130$ & 140 \\
\hline $\mathrm{B} 2$ & & & 0.149 & 1.61 & 7 & 50 \\
\hline $\mathrm{C}$ & 0.259 & 0.769 & & & & \\
\hline $\mathrm{C} 1$ & & & 0.260 & 0.770 & $>150$ & $>150$ \\
\hline $\mathrm{C} 2$ & & & 0.267 & 0.791 & 36 & 60 \\
\hline
\end{tabular}

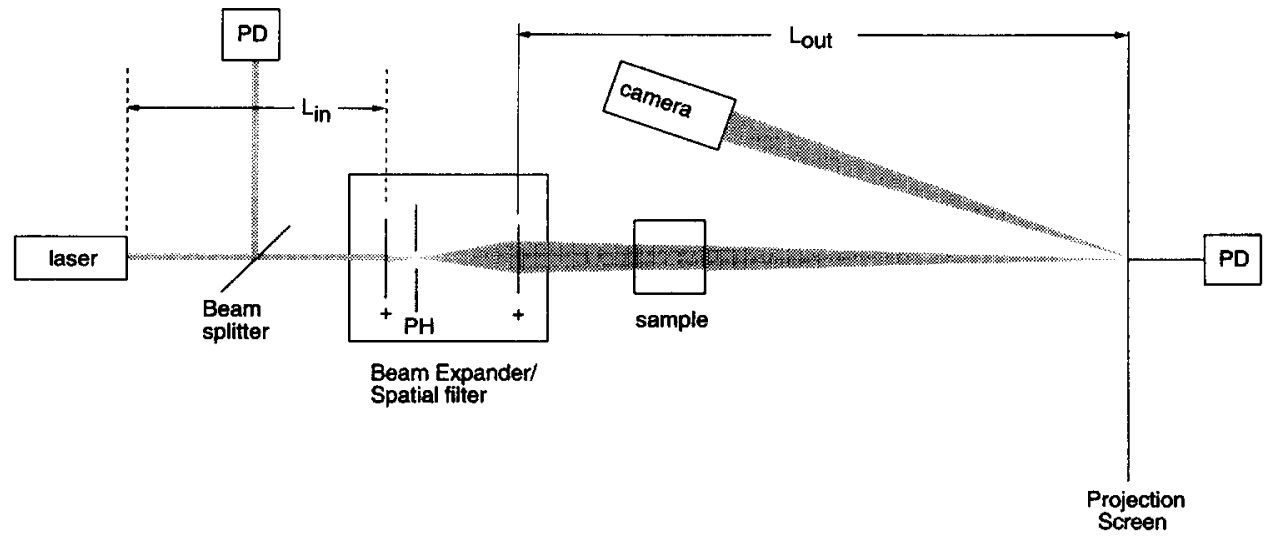

Fig. 2. Small angle light scattering apparatus. $\mathrm{PD}=$ photodiode and $\mathrm{PH}=$ pinhole.

projection screen made from paper. We have assumed that the intensity is linearly scattered from this screen. An additional pinhole $(6 \mathrm{~mm})$ between the spatial filter and the sample limited the laser beam near its first diffraction minimum. The light scattering pattern at the projection screen was not disturbed by the primary laser beam which passed through a hole (diameter $5 \mathrm{~mm}$ ) in the screen. The SALS pattern was recorded by a CCD-camera (Electrim, Princeton, $\mathrm{NJ}$ ) with a resolution of $165 \times 192$ pixels and 8 bit accuracy. The exposure time was $100 \mathrm{~ms}$, and adjustment of the camera diaphragm prevented overflow. Scattering angles were calibrated by imaging light diffracted by a grating (100 lines $/ \mathrm{mm})$. The accessible range of scattering angles was between $0.4^{\circ}$ and $8.1^{\circ}$, corresponding to wave vectors between 100 and $2000 \mathrm{~mm}^{-1}$. Structure in the light scattering pattern related to individual particles is 
expected at a wave vector of the order of $2 \times 10^{5} \mathrm{~mm}^{-1}$, which is far beyond the accessible range. The $2 \mathrm{D}$ images recorded by the $\mathrm{CCD}$-camera were radially averaged, resulting in arrays of intensity versus wave vector. Images could be collected and stored each $250 \mathrm{~ms}$.

During an experiment a series of images was collected and the sample transmission was measured with a photodiode. The time series was corrected for dark current and stray light by substracting an early image. Intensities were corrected for the increasing turbidity by dividing by transmission [47].

\subsection{Light microscopy}

The phase separating mixtures were studied using a Zei $\beta$ axioplan microscope in transmission with a $20 \times$ objective. The precise magnification was calibrated by imaging a diffraction grating (100 lines $/ \mathrm{mm})$ with the microscope. The systems were also studied with a Leitz Orthoplan polarizing microscope including a video camera. The characteristic length scale of the phase separating system could be determined from a 2-D Fourier transform of a digitized micrograph.

\section{Results}

\subsection{Visual observations}

After homogenization of the system in the two-phase region, the samples rapidly became turbid, indicating the onset of the unmixing process. A few minutes later upward and downward streaming of droplets could be observed as vertical stripes in the system, probably due to sedimentation and creaming of colloid-rich and colloid-poor domains because of the density difference between these domains. It took about half an hour for the samples to become less turbid at the top and bottom, and a rather clouded interface was observed. Finally, after approximately $1 \mathrm{~h}$ the system consisted of two transparent phases, separated by an interface which remained sharp and fluid over months.

\subsection{Small angle light scattering}

SALS could only be used to study the first few minutes of the phase separation process due to the decreasing transmission and the influence of gravity which deforms the circular scattering pattern. During this time a typical scattering ring could be observed at small wave vector $(q)$, with a maximum (at wave vector $q_{\max }$ ) that, from the start of the measurement, increased in intensity and moved to smaller wave vector (Fig. 3). This indicates that there is a characteristic length scale in the system, which increases in time. Finally, this ring merges into strong forward scattering.

After homogenization, transmission of the unmixing samples rapidly decreased. Table 2 gives the time $t_{10}$ after which transmission becomes smaller than $10 \%$ for the 
various mixtures. Unless stated otherwise, light scattering curves with transmission smaller than $10 \%$ have been omitted from analysis because multiple scattering is considered to be too important. Table 2 also gives the time $t_{\mathrm{g}}$ after which the circular scattering pattern changed into an ellipsoidal shape, due to gravity-induced streaming of colloid-rich and colloid-poor domains. From then on radial averaging is not allowed anymore. The influence of gravity was particularly pronounced for sample A. For all samples an increase in supersaturation leads to an increase of the phase separation rate. For experiments closest to the phase boundary the scattering ring can
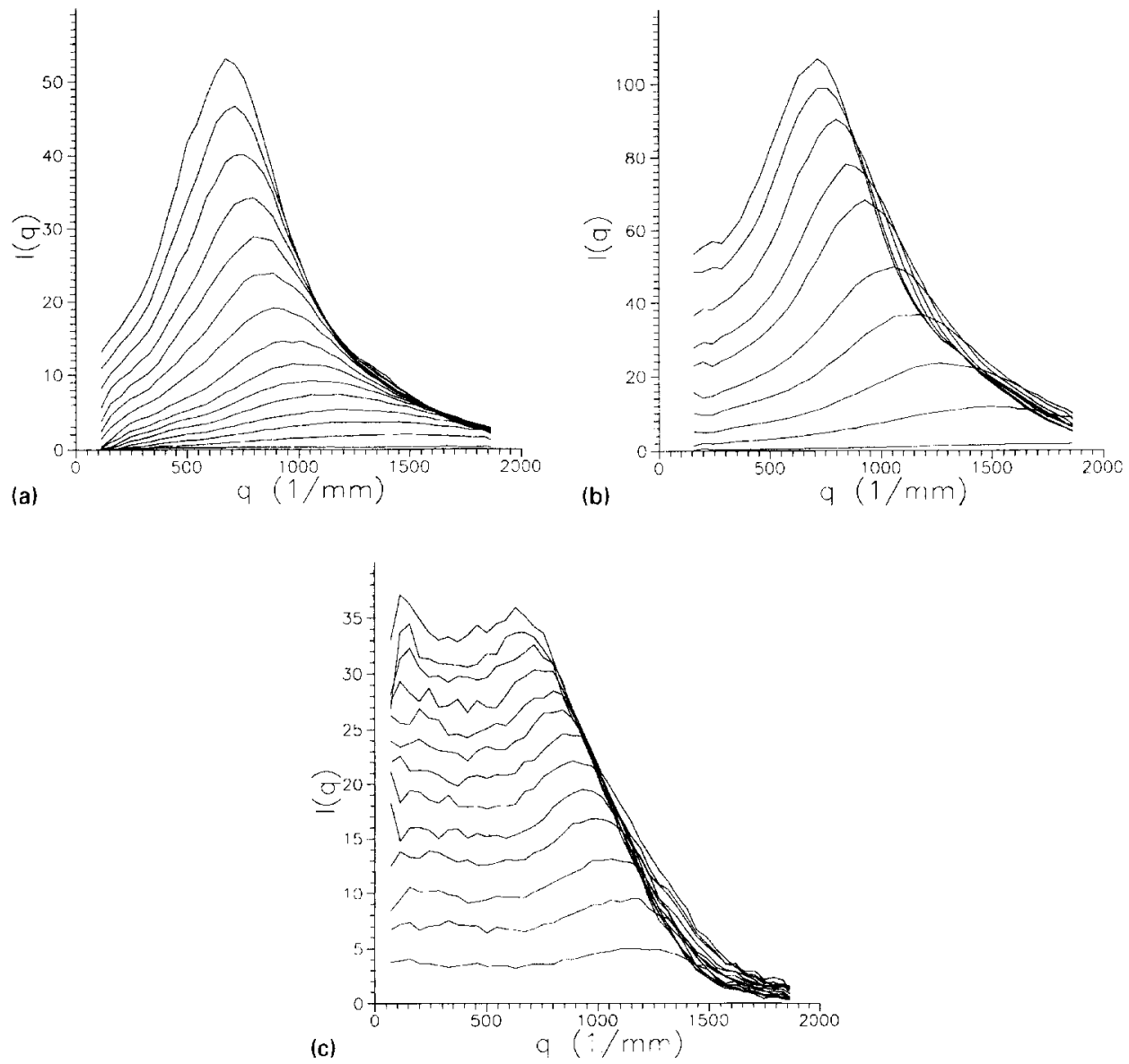

Fig. 3. Sequences of scattering intensities (corrected for transmission) as function of wave vector for several experiments. The scattering curves show a peak which increases in intensity and shifts to smaller wave vector in time. For experiment $A_{1}$ the final time is $45 \mathrm{~s}$ and the time interval between curves about $3 \mathrm{~s}$ (a). For experiment $A_{2}$ the final time is $20 \mathrm{~s}$ and the time interval between curves about $2 \mathrm{~s}$ (b). For experiment $\mathrm{A}_{3}$ the final time is $2.6 \mathrm{~s}$ and the time interval between curves about $0.2 \mathrm{~s}$ (c). For experiment $\mathrm{B}_{1}$ the final time is $130 \mathrm{~s}$ and the time interval between curves about $10 \mathrm{~s}(\mathrm{~d})$. For experiment $\mathrm{B}_{2}$ is the final time $18 \mathrm{~s}$ and the time interval between curves about $2 \mathrm{~s}(\mathrm{e})$. For experiment $\mathrm{C}_{1}$ the final time is $145 \mathrm{~s}$ and the time interval between curves about $15 \mathrm{~s}(\mathrm{f})$. For experiment $\mathrm{C}_{2}$ the final time is $50 \mathrm{~s}$ and the time interval between curves about $4 \mathrm{~s}(\mathrm{~g})$. 

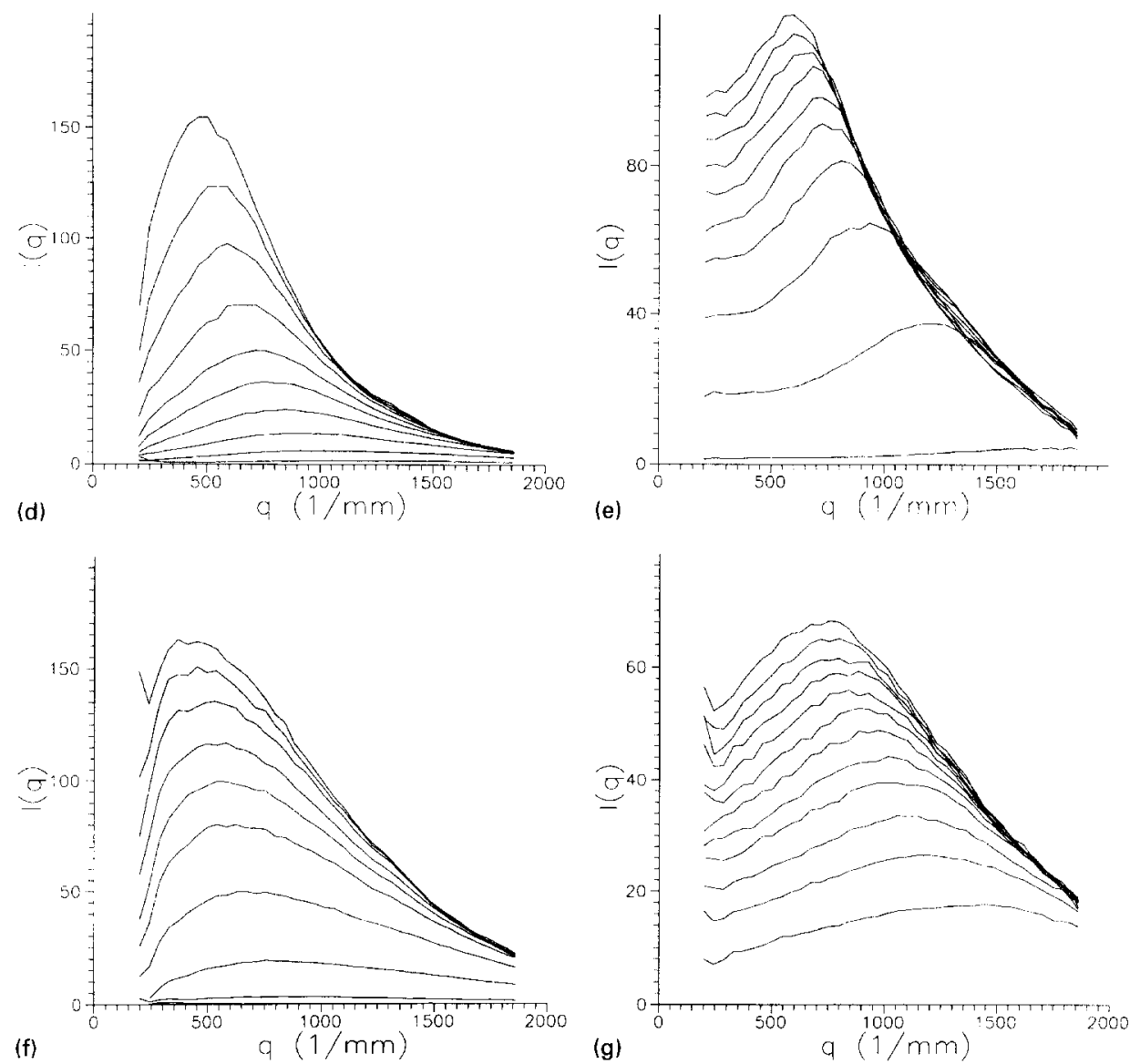

Fig. 3. Continued.

be observed visually on the projection screen at a large scattering angle before it is detected by the CCD-camera. We have not optimized the setup further in order to study this high wave vector range.

\subsection{Light microscopy}

Light microscopy allows direct imaging of the time evolution of the structure in an unmixing system. In our case an advantage of light microscopy is that the accessible measuring time is much longer than for light scattering, which is hampered by multiple scattering and by gravity effects.

The time evolution in experiment $A_{1}$, which is close to the phase boundary, was studied during the first minutes of phase separation. Immediately after homogenization, a bicontinuous structure of darker and lighter domains of the type demonstrated in Fig. 4(a) (14 s after homogenization) was observed. In the following figures (Figs. 4(b)-(d)) it can be seen that the structure coarsened with time. After about $150 \mathrm{~s}$ 


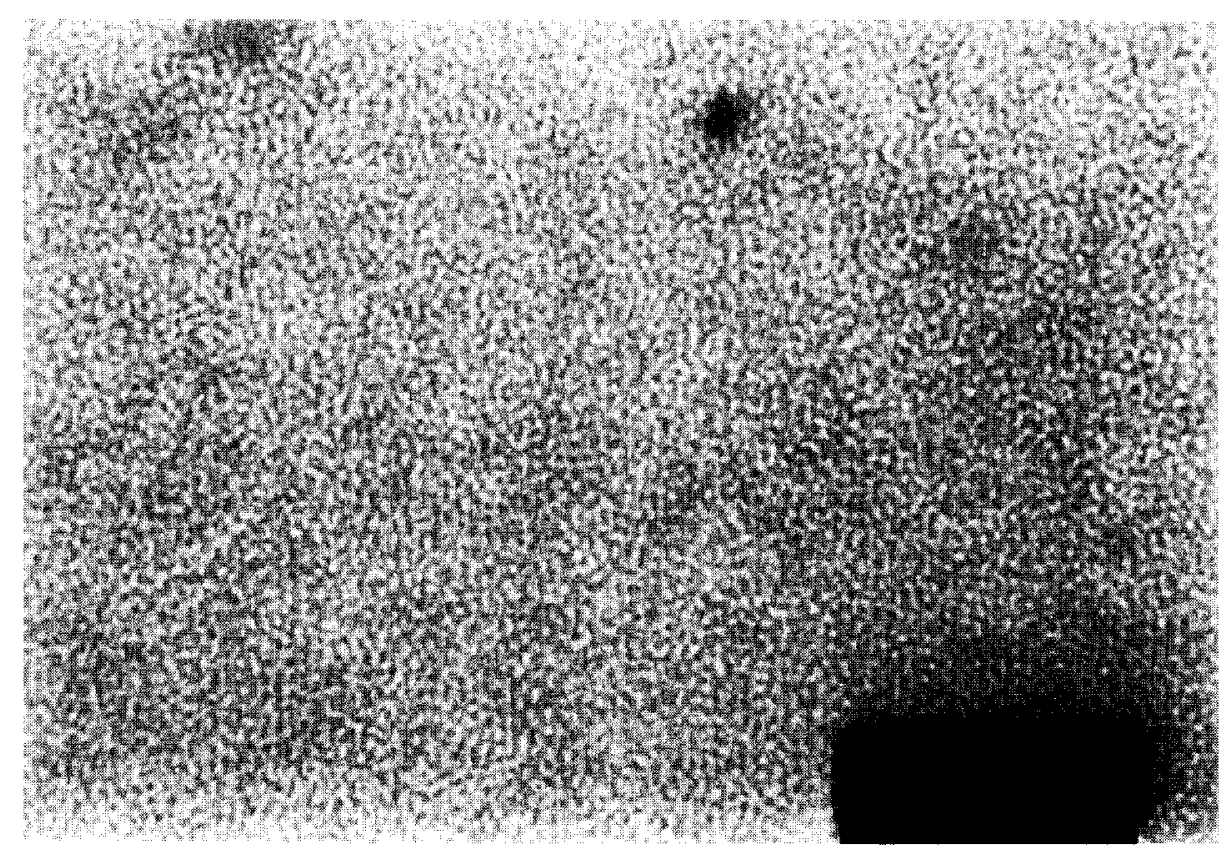

a)

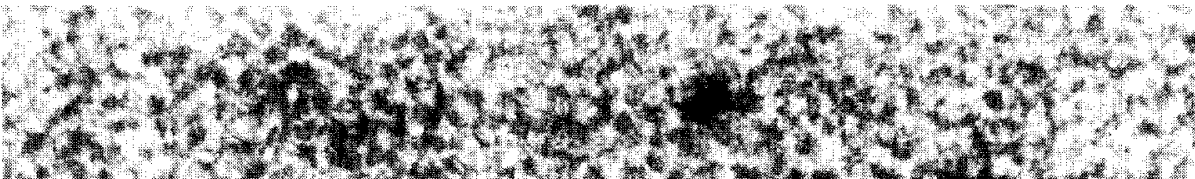

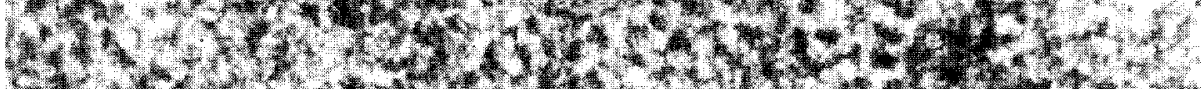

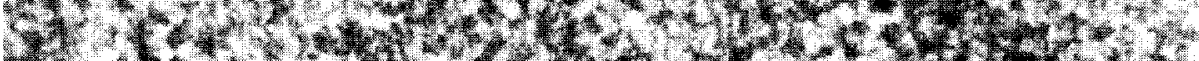

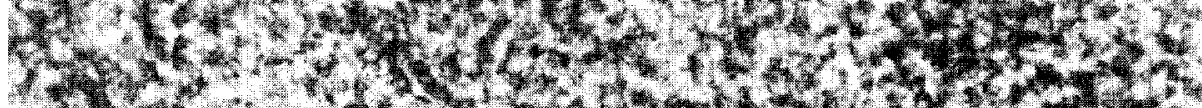

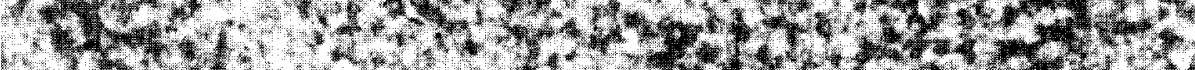

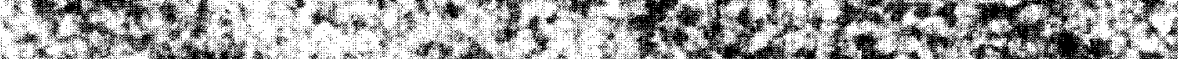

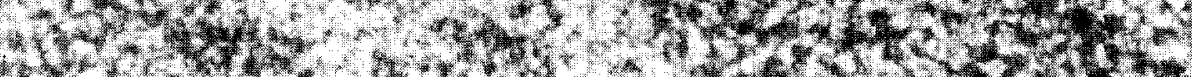

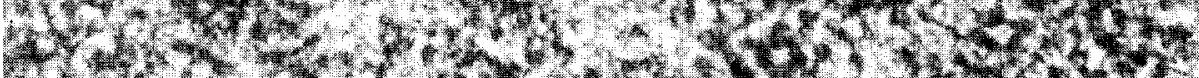

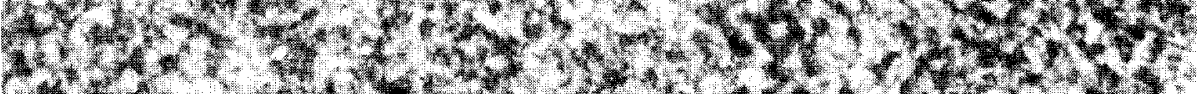

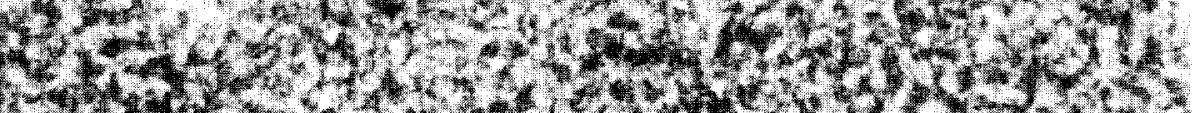

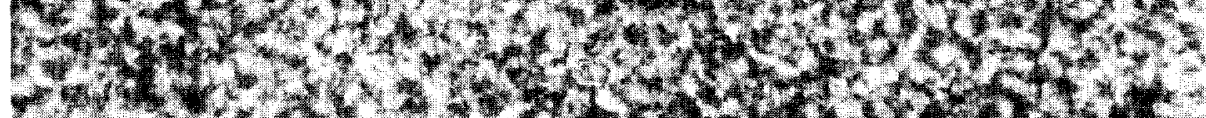

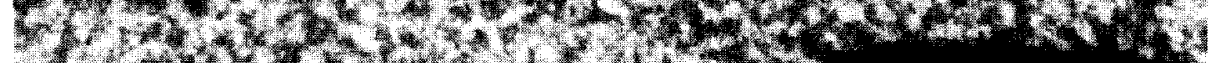

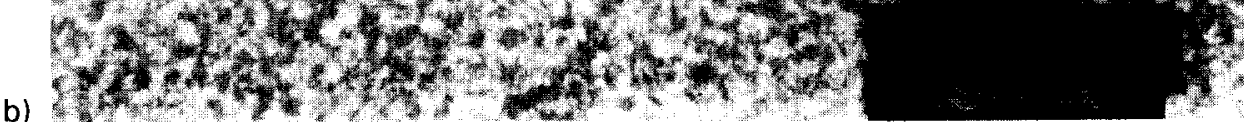

Fig. 4. Micrographs of sample $A$ in experiment $A_{1}$ at a sequence of times after homogenization (a) $14 \mathrm{~s}$, (b) $40 \mathrm{~s}$, (c) $70 \mathrm{~s}$, (d) $100 \mathrm{~s}$. The bar corresponds to $100 \mu \mathrm{m}$. 


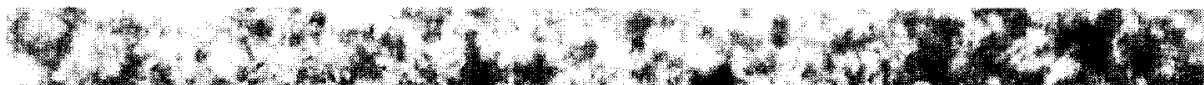

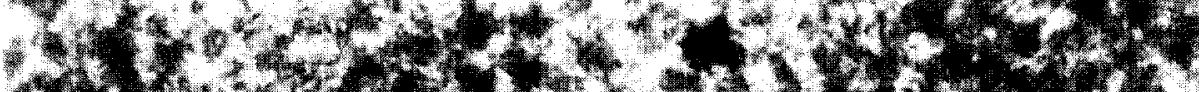

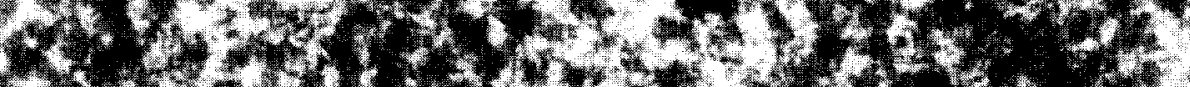

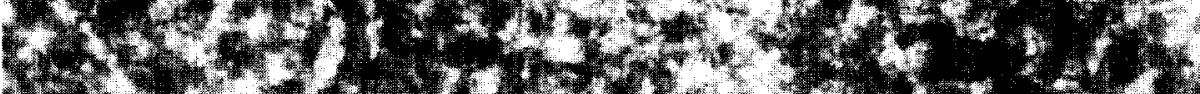

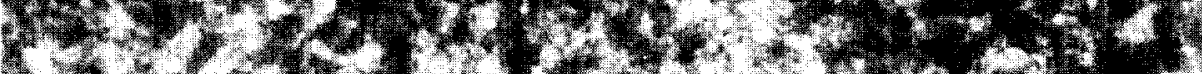

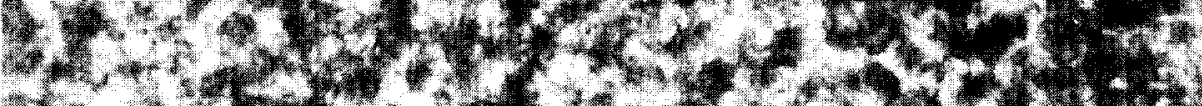

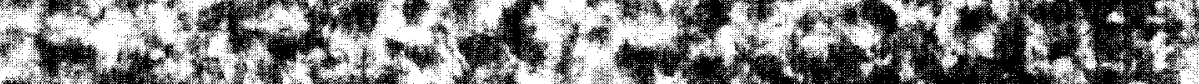
H.

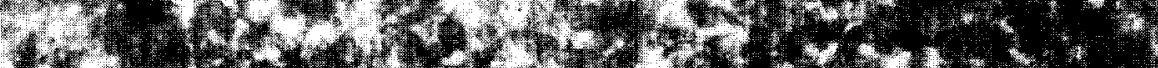

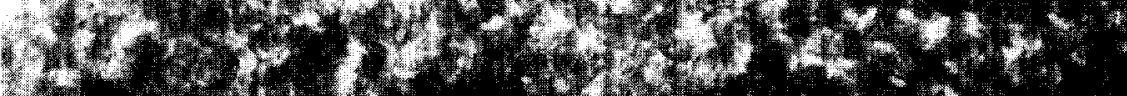

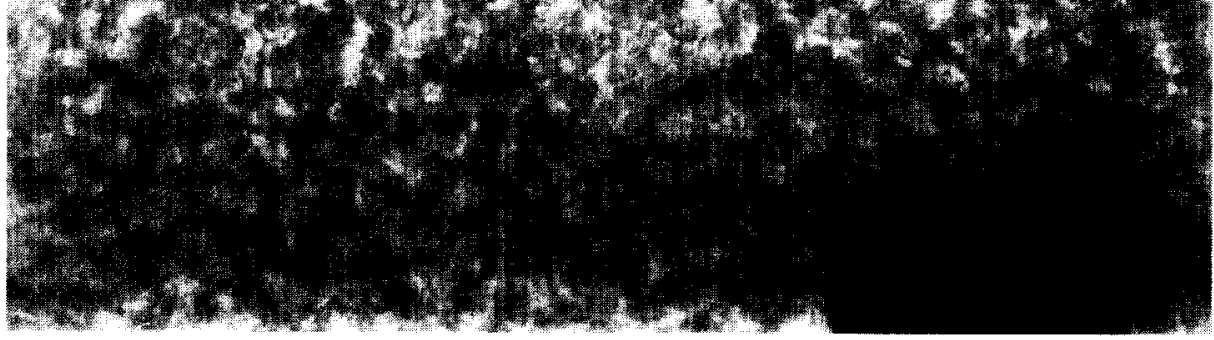

c)

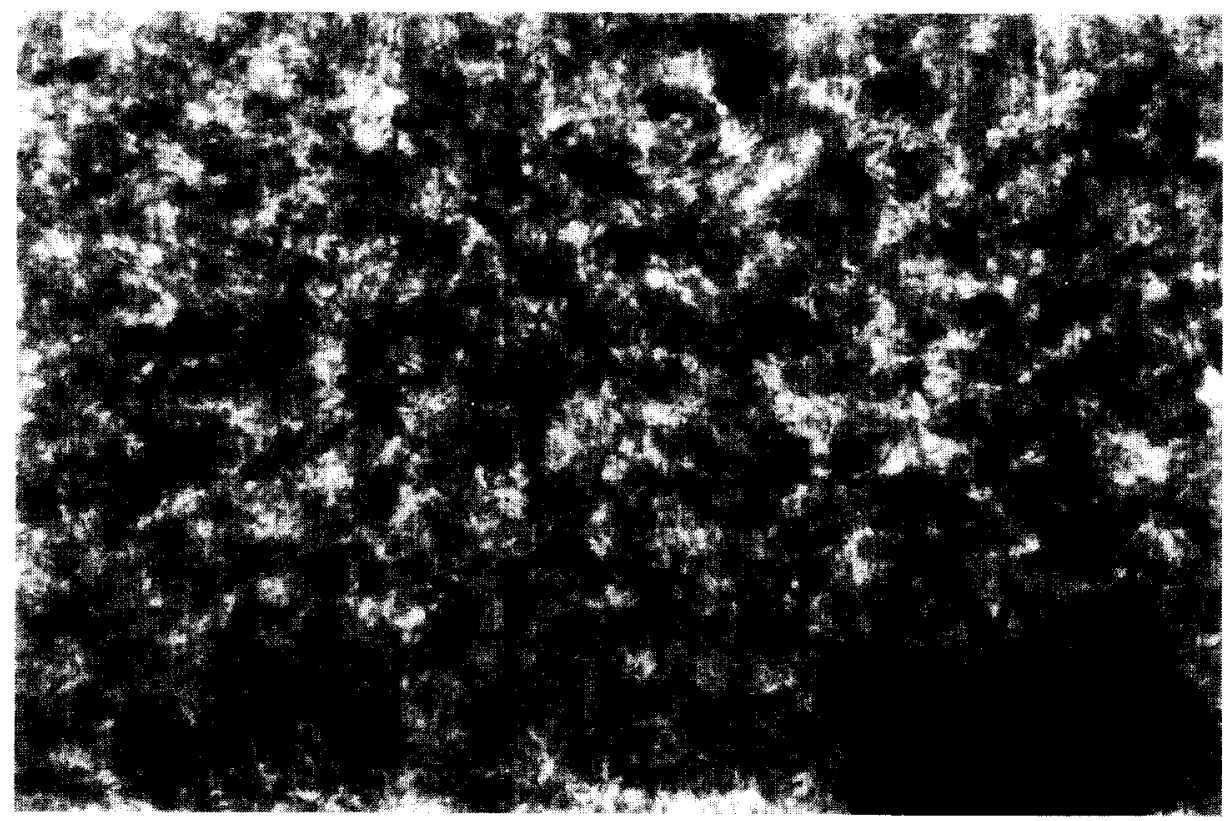

Fig. 4. Continued. 
streaming was observed, probably because of flow of phase separating domains due to gravity. The structures observed in experiment $A_{2}$ and $A_{3}$ were very similar as shown in Fig. 4 except that the time evolution was considerably faster.

Sample B (more polymer, see Fig. 1) at small supersaturation, initially behaved roughly like sample A (Fig. 5(a); $74 \mathrm{~s}$ after homogenization). A bicontinuous structure was formed immediately and the domains coarsened with time. After a few minutes however small droplets could be observed in the bulk of the dispersion (Fig. 5(b): $200 \mathrm{~s}$ and Fig. 5(c): $362 \mathrm{~s}$ after homogenization). They grew larger but they kept their spherical shape and did not seem to coalesce. This droplet formation has not been observed in sample A.

Initially sample $\mathrm{C}$ (more colloid, see Fig. 1) at small supersaturation, showed no clear structure. Slowly a pattern developed, which immediately looked more like small droplets instead of the bicontinuous structure observed for samples A and B (Fig. 6(a): $45 \mathrm{~s}$ after homogenization). The droplets grew larger, first mainly by spontaneous addition of material and later also by coalescence of colliding droplets (Fig. 6(b); $410 \mathrm{~s}$ after homogenization). Although these droplets were also visible in the bulk, it was clear that they preferentially grew at the upper glass wall, finally yielding a film at which smaller droplets from the bulk coalesced (Fig. 6(d); $600 \mathrm{~s}$ after homogenization). Since the polymer-rich minority phase is expected to evolve as upper phase, we suppose that the droplets consist of polymer-rich phase. At the lower glass wall no droplets can be discerned. In sample A and B we have not observed such a strong preference for the upper glass wall.

\section{Analysis and discussion}

\subsection{Phase diagram}

By comparing Fig. 1 with predictions based upon the statistical mechanical theory for phase separation in colloid-polymer mixtures [18] we found that the experimental phase boundary lies considerably above the calculated one. This is not surprising as the polymer volume fraction exceeds the overlap concentration indicating that the polymers penetrate each other (semi-dilute regime). This is not taken into account by the theory [18]. Similar deviations from theory were found by Patel et al. [13] and by Ilett et al. [2].

\subsection{Small angle light scattering}

The pronounced peak in the light scattering curves for our unmixing mixtures (Fig. 3) is strongly reminiscent of the light scattering in phase separation studies in binary liquids and polymer mixtures. This peak shifts to smaller $q$-values in time, and simultaneously the overall intensity increases. The accessible measuring time is limited by a decrease in transmission to the first minutes of phase separation. 


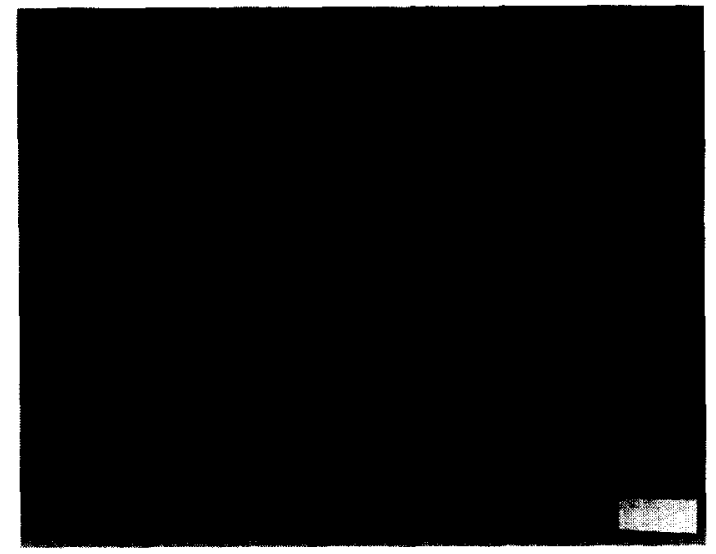

b)

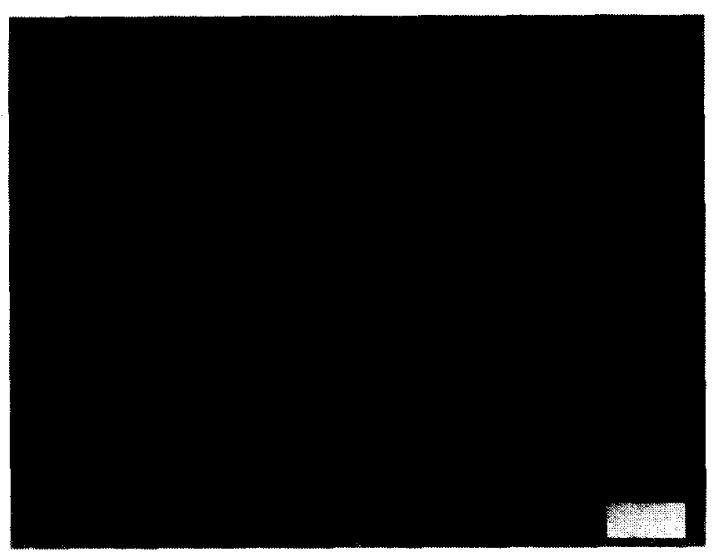

c)

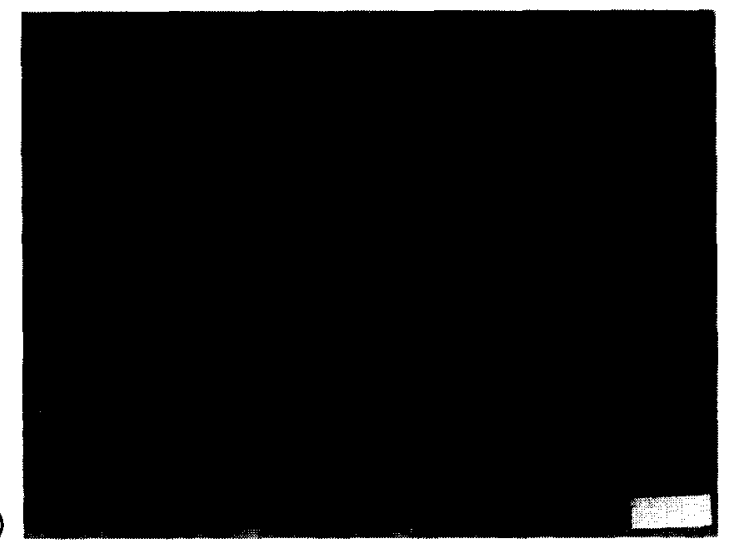

Fig. 5. Micrographs of sample $B$ close to the phase boundary (comparable to $B_{1}$ ) at a sequence of times after homogenization (a) $74 \mathrm{~s}$, (b) $200 \mathrm{~s}$, (c) $362 \mathrm{~s}$. The bar is $40 \mu \mathrm{m}$. 
a)

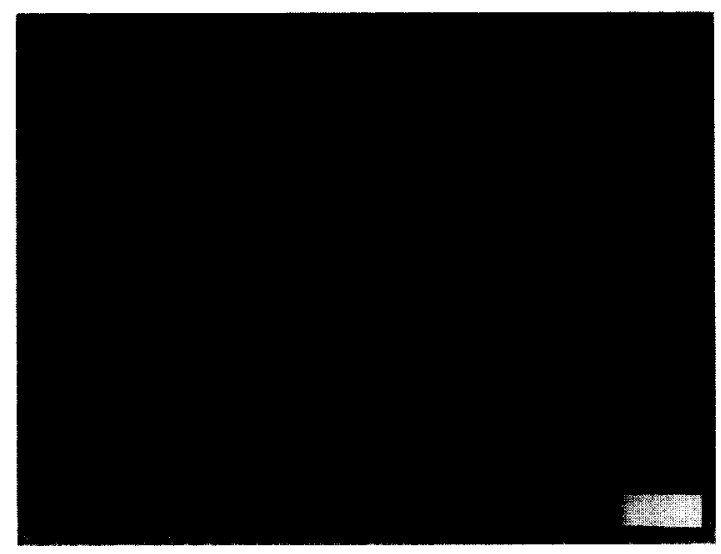

b)

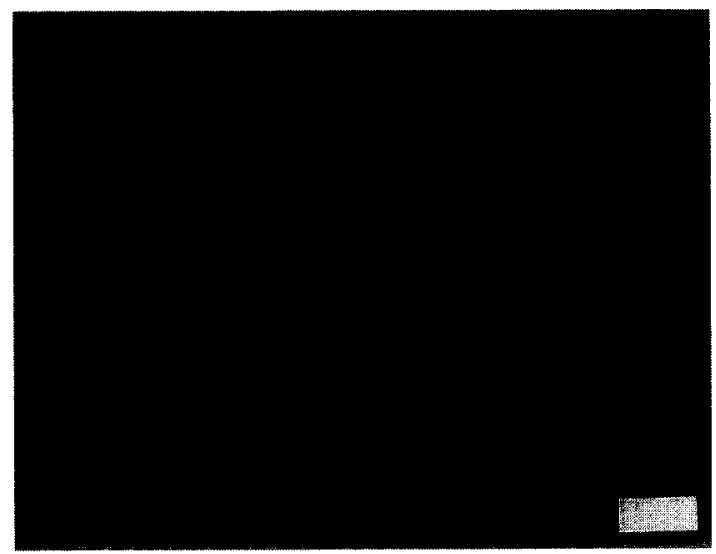

c)

Fig. 6. Micrographs of sample $\mathrm{C}$ close to upper cuvette wall (comparable to $\mathrm{C}_{1}$ ) at a sequence of times after homogenization (a) $45 \mathrm{~s}$, (b) $410 \mathrm{~s}$, (c) $600 \mathrm{~s}$. The bar is $40 \mu \mathrm{m}$. 
For samples with a relatively high supersaturation the accessible time scale is even shorter.

A noticeable feature is the fact that for the samples closest to the phase boundary (sample $A$ in experiment $A_{1}$, sample $B$ in experiment $B_{1}$ and the sample $C$ in experiment $C_{1}$ ) the intensity at small wave vectors drops to low values, while for samples with higher supersaturation $\left(A_{3}, B_{2}\right.$ and $\left.C_{2}\right)$ it rises substantially. A possible explanation for this difference might be the effect of multiple scattering for the samples with higher supersaturation. However, a tentative correction for multiple scattering showed that multiple scattering could only account for part of the upswing at small wave vector. Alternatively, one might speculate that the more highly supersaturated samples become heterogeneous in length scales, leading to a washing out of the structure factor.

The appearence of a peak in the light scattering pattern is commonly interpreted as an indication of spinodal decomposition, which in the early stages is described by the linear Cahn theory. This will be investigated in Section 4.2.1. The shift of the peak to smaller wave vectors indicates a coarsening of the characteristic length scale in the system, which will be analyzed in Section 4.2.2. Finally, we check whether the experimental data show dynamic scaling behavior (Section 4.2.3).

\subsubsection{Early stage}

Initial stages of spinodal decomposition are commonly analyzed within a framework of Cahn-Hilliard theory $[25,32,34]$ that predicts that there is a fastest-growing density mode which gives rise to a ring in the small angle light scattering pattern. Such a fastest-growing mode arises because there is a competition between growth of small-wavelength modes for which particles only have to diffuse over short distances, and growth of long-wavelength modes with shallow density gradients, for which the thermodynamic driving force is favourable.

Cahn derived an equation of motion for the concentration in a thermodynamically unstable system. This equation implies exponential growth of concentration waves with long enough wavelength during the initial stage of the phase separation. This leads to the following prediction for the light scattering intensity:

$$
I(q, t) \propto \exp [2 R(q) t]
$$

where the amplification factor $R(q)$ is given by

$$
R(q)=D_{\text {eff }} q^{2}\left[1-\frac{1}{2}\left(\frac{q}{q_{0}}\right)^{2}\right] .
$$

The fastest growing mode for which $q$ equals $q_{0}$ produces a peak in the scattering pattern at wave vector $q_{0}$.

Although experimentally we detected a scattering ring with a continuously decreasing radius (Fig. 3), we attempted like other authors [26], to find a linear Cahn-regime in the initial stage of phase separation. Fig. 7 shows the logarithm of the scattered 


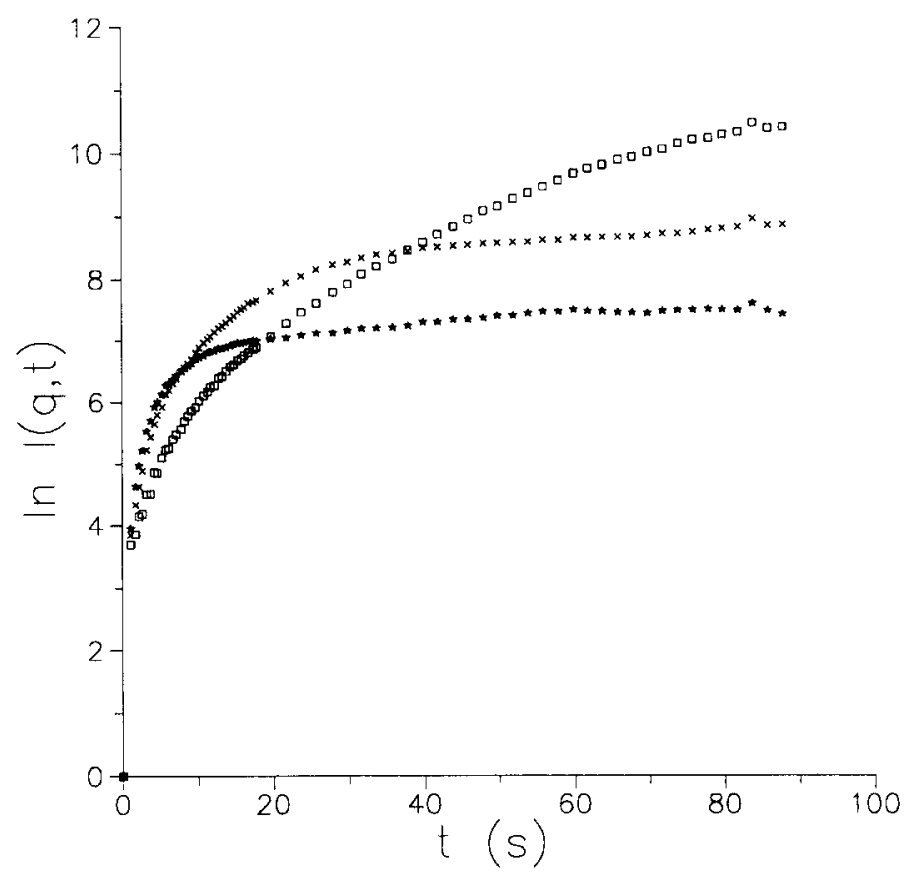

Fig. 7. Logarithm of intensity versus time for sample $\mathrm{A}$ in experiment $\mathrm{A}_{1}$. squares: $q=500 \mathrm{~mm}^{-1}$, crosses: $q=1015 \mathrm{~mm}^{-1}$, stars: $q=1789 \mathrm{~mm}^{-1}$. From the initial slope the amplification factor $R(q)$ is derived based upon a linear fit.

intensity versus time for experiment $A_{1}$ for several wave vectors. In all cases, intensity is initially seen to rise, before it reaches a saturation value. Saturation is reached earlier for larger wave vectors. Although in most cases the initial linear relation between logarithm of intensity and time is doubtful, we derived the $q$-dependent $R$ from the initial slope (Eq. (1)) by performing a linear fit through the first data points. In Fig. 8 we present the 'Cahn plot', i.e. $R(q) / q^{2}$ versus $q^{2}$, for sample $\mathrm{A}$ in experiment $\mathrm{A}_{1}$. It is evident that we do not obtain a linear relation between $R(q) / q^{2}$ versus $q^{2}$ predicted by the linear Cahn theory. For the early stage unmixing in the other mixtures $\mathrm{B}$ and $\mathrm{C}$ a similar deviation from the Cahn plot was found.

The obtained curvatures in the Cahn plot of Fig. 8 are very much alike other experimental results [27, 29,48]. Binder discussed a modified Cahn-analysis [49], in which additional time-dependent modes are taken into account, and which resembles the observed curvatures.

Apparently, our experimental data cannot be described by linear Cahn theory on the earliest accessible time scales in the unmixing colloid-polymer mixtures, which might indicate that either the initial stage of phase separation is completed too soon to be detected for our samples, or vigorous shaking does not produce a fully homogenized state. 


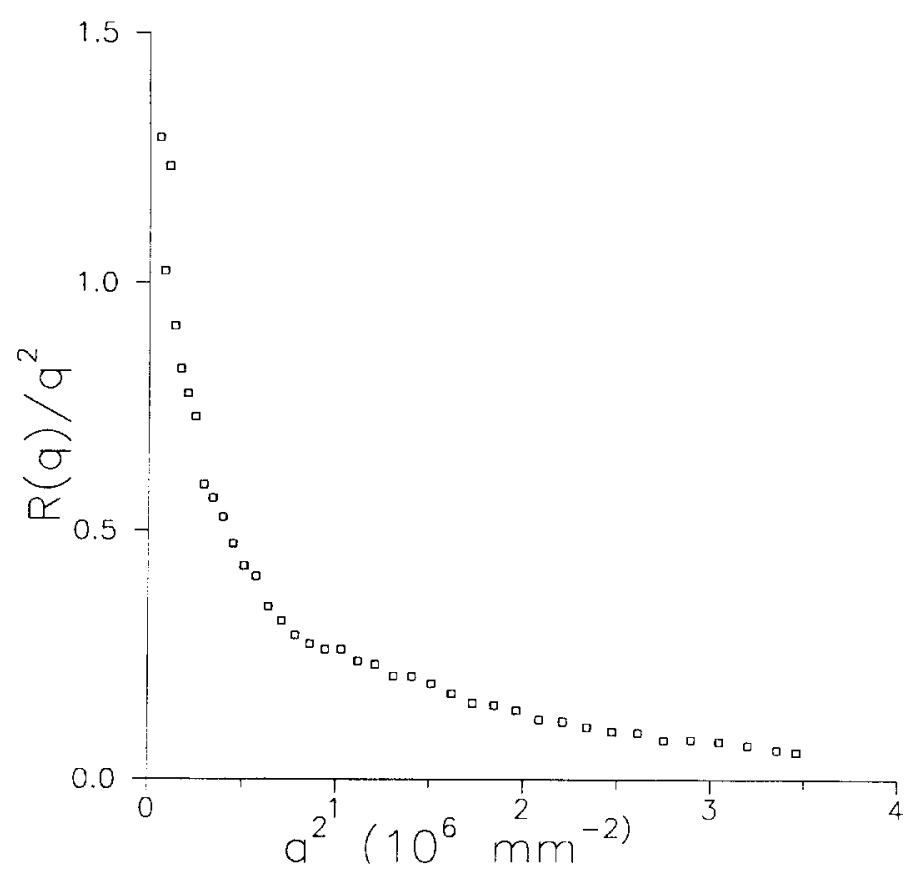

Fig. 8. Cahn plot for experiment $A_{1}$.

\subsubsection{Coarsening behavior}

Following Siggia [41] it has become customary to distinguish three stages of domain growth: in the first diffusive growth stage the domain size $l$ satisfies $l \propto t^{1 / 3}$. The corresponding coarsening mechanisms can either be the evaporation and condensation of particles from smaller to larger domains $[39,40]$, or the diffusion and coalescence of domains [38]. In the flow stage for more concentrated mixtures coarsening becomes hydrodynamically controlled and $l \propto t$ [41]. Finally, we reach the gravitational stage, when $l$ is larger than the capillary length $l_{\text {cap }}=\sqrt{(\sigma / g \Delta \rho)}$, where $\sigma$ is the interfacial tension, $g$ the gravitational acceleration and $\Delta \rho$ the density difference between the two co-existing phases.

By plotting $q_{\max }$ versus $t$ on a log-log scale (Fig. 9) we can derive the characteristic growth exponent. We note again that for all experiments we limit ourselves to times smaller than $t_{\mathrm{g}}$ or $t_{10}$ (as defined in Section 3.2), whichever is smaller. It appears that for all samples the change of $q_{\max }$ with time can, at least initially, be described by a power law with an exponent close to $-\frac{1}{3}$, indicative of diffusive growth [41]. This exponent does not discriminate between a mechanism of evaporation of individual particles from small domains and condensation onto larger domains on the one hand and diffusion and coalescence of domains on the other hand. However, since Siggia [41] states that for volume fractions of minority phase larger than $1 \%$ the diffusion and coalescence mechanism becomes dominant, we assume that our coarsening is mostly described by this scenario. In those cases where the measuring time exceeds 

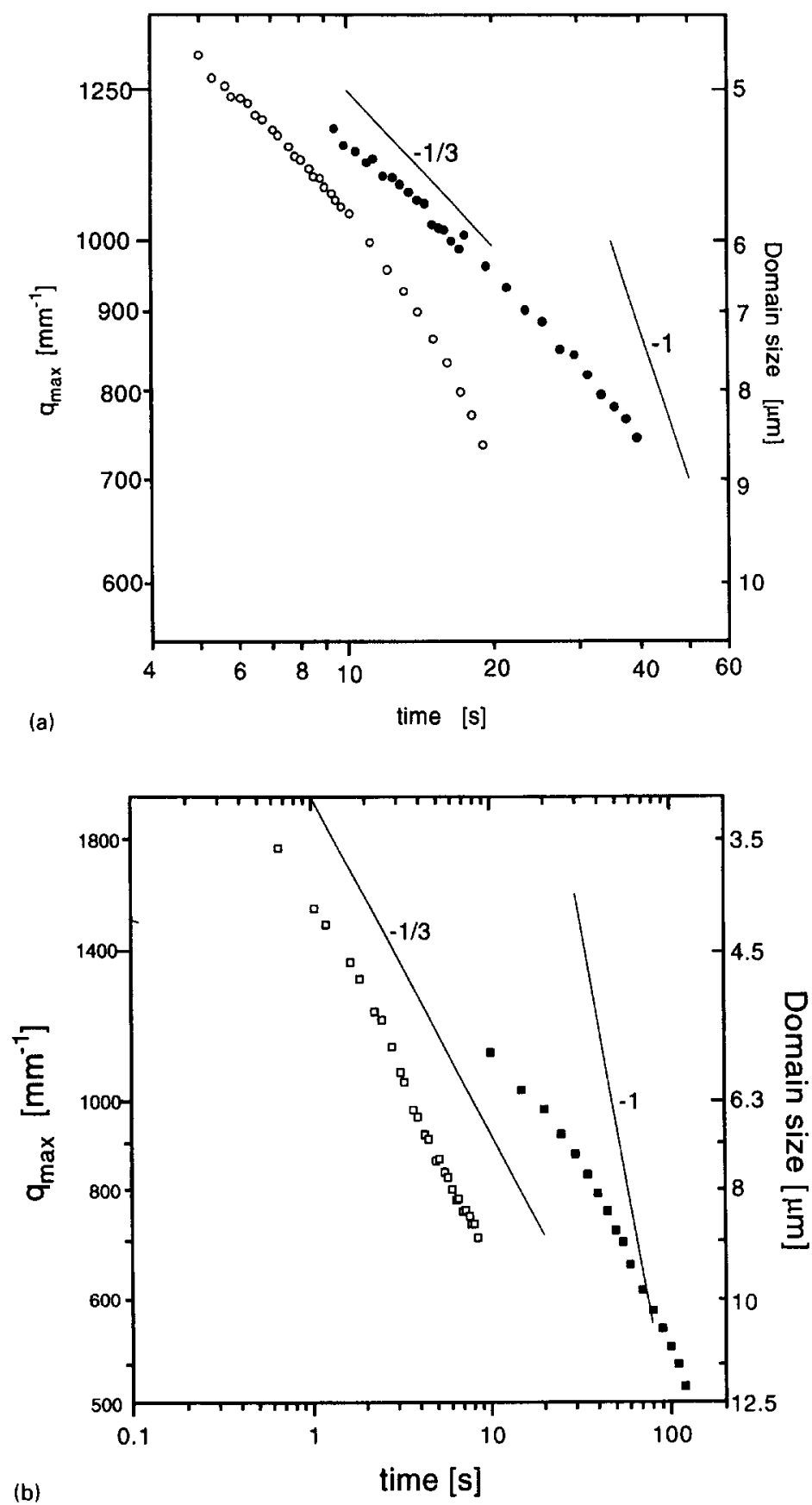

Fig. 9. Double logarithmic plot of $q_{\max }$ versus time (a) for sample $\mathrm{A}$ in experiment $\mathrm{A}_{1}$ (filled circle) and $\mathrm{A}_{2}$ (open circle); (b) for sample $B$ in experiment $B_{1}$ (filled square) and $B_{2}$ (open square) and (c) for sample $C$ in experiment $C_{1}$ (filled triangle) and $C_{2}$ (open triangle). Characteristic exponents for coarsening behavior dominated by diffusion and coalescence $\left(-\frac{1}{3}\right)$ [37] and by hydrodynamic flow $(-1)$ [40] are added. The domain size which is the reciprocal of $q_{\max }$ has been included along the right axis. 


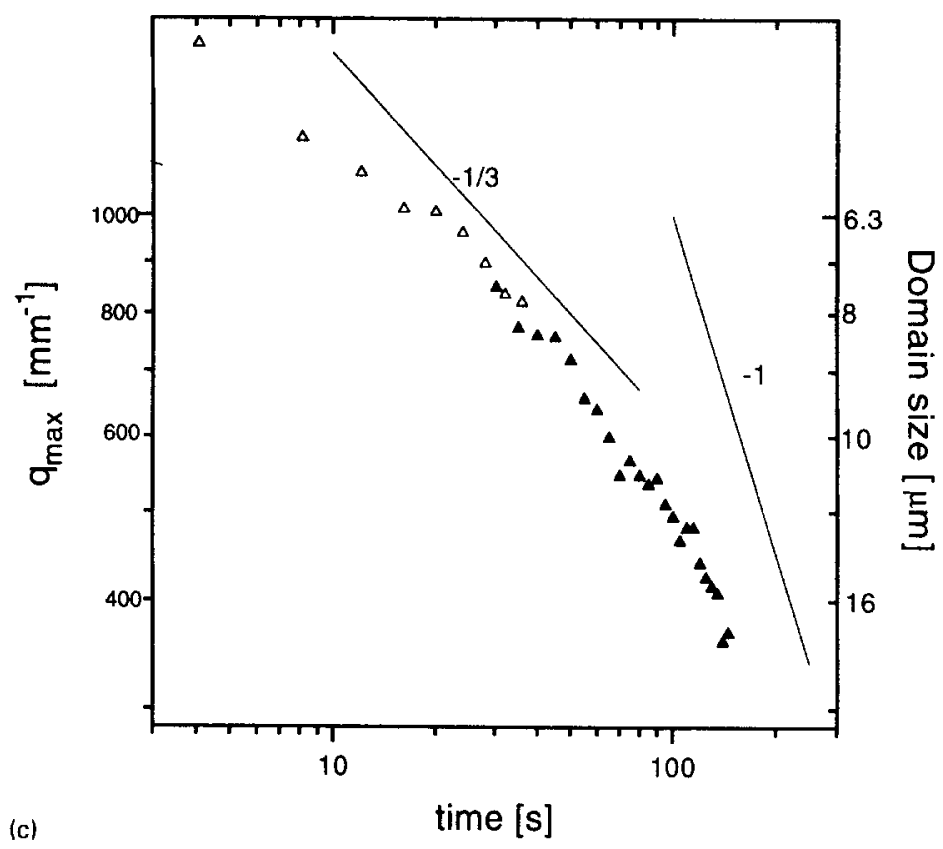

Fig. 9. Continued.

$100 \mathrm{~s}$ some speeding up of coarsening kinetics is observed, but no clear cross-over to hydrodynamically controlled coarsening $\left(q_{\max } \propto t^{-1}\right)$ can be detected.

In order to estimate when coarsening will be dominated by gravity-induced motion [41], we need a value for $l_{\text {cap }}$. Using a $\sigma$ equal to $4 \times 10^{-6} \mathrm{~N} \mathrm{~m}^{-1}$ (based upon an analytical expression by Kalikmanov et al. [50] in which we applied a depletion potential) and a $\Delta \rho$ equal to $1 \times 10^{3} \mathrm{~kg} \mathrm{~m}^{-1}$, we obtain a $l_{\text {cap }}$ equal to $20 \mu \mathrm{m}$. This corresponds to a wave vector $q_{\text {cap }}=2 \pi / l_{\text {cap }}$ of $3 \times 10^{2} \mathrm{~mm}^{-1}$. By extrapolating the data points for the off-critical experiments $B_{1}, B_{2}$ and $C_{1}$ in Figs. $9(b)$ and (c) we can deduce time $t_{\text {cap }}$ for which $q$ equals $q_{\text {cap }}$. We find that for the experiments $\mathrm{B}_{1}, \mathrm{~B}_{2}$ and $\mathrm{C}_{1}$ the extrapolated value of $t_{\text {cap }}$ is of the same order of magnitude as the experimentally determined $t_{\mathrm{g}}$ (Table 2). For experiments $\mathrm{A}_{1}$ and $\mathrm{A}_{2}, t_{\text {cap }}$ based upon extrapolation is much larger than $t_{\mathrm{g}}$ indicating that $\sigma$ in the critical mixture $\mathrm{A}$ is probably smaller than the estimated $4 \times 10^{-6} \mathrm{~N} \mathrm{~m}^{-1}$.

\subsubsection{Dynamic scaling}

For many phase separating structures dynamic scaling is found, i.e. the evolution of the morphology can be described using only one time-dependent length scale. Binder $[35,38]$ predicted upon a theoretical basis, and Marro, Lebowitz and Kalos [42, 43] found from simulation results that the structure factor $S(q, t)$ obeys a simple scaling relation:

$$
S(q, t)=q_{\max }^{-3} F\left(q / q_{\max }\right),
$$


where $q_{\max }$ is the time-dependent wave vector at which the peak maximum is found, $t$ the time since the quench and $F$ a universal scaling function.

Applying Eq. (3) to the scattering intensity $I(q, t)$, which is directly proportional to the structure factor $S(q, t)$ since the form factor at these small wave vectors is considered to be equal to 1 for these small particles, we found that our scattering data do not satisfy the scaling relation given by Eq. (3). If we normalize our data by $\int I(q, t) q^{2} d q$ as was suggested by $[23,27,43]$, we find dynamical scaling in a number of cases. The scaling relation is then given by

$$
\frac{I(q, t) q_{\max }^{3}}{\int I\left(q^{\prime}, t\right) q^{\prime 2} d q^{\prime}}=F\left(q / q_{\max }\right)
$$

The scaling relation (4) can be understood as follows [51]. When inhomogeneities of the macroscopic colloid number density $\delta p(\boldsymbol{r}, t)=\rho(\boldsymbol{r}, t)-\bar{\rho}$ with respect to the average value $\bar{\rho}=N / V$ are sufficiently developed during the unmixing process, their structure factor for $q<q^{*}=\pi / r_{\mathrm{c}}$, where $r_{\mathrm{c}}$ represents the radius of the colloid, is given by

$$
S(q, t)=\frac{1}{N}\left\langle|\delta \rho(q, t)|^{2}\right\rangle_{\text {init }}
$$

Here $\delta \rho(\boldsymbol{q}, t)$ is the Fourier transform of $\delta \rho(\boldsymbol{r}, t)$ and $\langle\cdots\rangle_{\text {init }}$ indicates the ensemble average with respect to initial conditions for the density. When at each instant of time there is a dominant length scale $\xi$, the ratio

$$
\frac{\left\langle\delta \rho(\boldsymbol{r}, t) \delta \rho\left(\boldsymbol{r}^{\prime}, t\right)\right\rangle_{\mathrm{init}}}{\left\langle(\delta \rho(\boldsymbol{r}, t))^{2}\right\rangle_{\mathrm{init}}} \equiv f\left(\frac{\left|\boldsymbol{r}-\boldsymbol{r}^{\prime}\right|}{\xi}\right)
$$

is only time-dependent through the time-dependence of $\xi$. The function $f(x)$, where $x=\left|\boldsymbol{r}-\boldsymbol{r}^{\prime}\right| / \xi$, defined by Eq. (6) is normalized to unity at $x=0$ for all times. Substitution of Eq. (6) into Eq. (5) leads to

$$
S(q, t)=\frac{4 \pi}{\bar{\rho}} \xi^{3}\left\langle(\delta \rho(\boldsymbol{r}, t))^{2}\right\rangle_{\text {init }} \int_{0}^{\infty} \frac{\sin (q \xi x)}{q \xi} f(x) x d x .
$$

Furthermore, integration of (5) with respect to $\boldsymbol{q}$ gives

$$
\left\langle(\delta \rho(r, t))^{2}\right\rangle_{\text {init }}=\frac{\bar{\rho}}{2 \pi^{2}} \int_{0}^{q^{*}} q^{\prime 2} S\left(q^{\prime}, t\right) d q^{\prime},
$$

where the integration over $q$ is limited to the $q$-range $q<q^{*}=\pi / r_{\mathrm{c}}$ relevant for the macroscopic unmixing process. The ensemble average $\left\langle(\delta \rho(\boldsymbol{r}, t))^{2}\right\rangle_{\text {init }}$ is the mean square amplitude of the variations in the number density, which increases as demixing proceeds. It is important to realize that the integration is only over the contribution to 
the structure factor that relates to the unmixing process, which is given by Eq. (5). Substitution of Eq. (8) into Eq. (7) finally leads to

$$
\frac{S(q, t) q_{\max }^{3}}{\int_{0}^{q^{*}} S\left(q^{\prime}, t\right) q^{\prime 2} d q^{\prime}}=16 \pi^{2} \int_{0}^{\infty} x \frac{\sin \left(2 \pi x q / q_{\max }\right)}{2 \pi q / q_{\max }} f(x) d x=F\left(q / q_{\max }\right),
$$

where $\xi=2 \pi / q_{\max }$. The right-hand side of Eq. (9) is only a function of $q / q_{\max }$ and can be identified as the scaling function $F\left(q / q_{\max }\right)$ in Eq. (4).

In Fig. 10 we plot the angular intensity distributions at different times during an experiment which have been scaled according to Eq. (4). In order to calculate $\int I(q, t) q^{2} d q$ we had to extrapolate our intensity measurements to $q$-values beyond the experimental accessible range $\left(100-2000 \mathrm{~mm}^{-1}\right)$. Since we do not know the precise shape of the scattering curves at large wave vector, we have limited ourselves to experiment $A_{1}, A_{2}, B_{1}$ and $B_{2}$ for which the light scattering at the largest experimentally accessible wave vectors already satisfied the Porod regime, so that we could extrapolate the intensity as $q^{-4}$ [52]. In the insets of Fig. 10 we show the time dependence of $\int I(q, t) q^{2} d q$, which is denoted by $S_{2} . S_{2}$ grows more or less proportionally with time, which means that, indeed, the mean square amplitude of the density variations increases. The scattering data obtained in experiment $A_{1}$ and $A_{2}$, scaled according to Eq. (4), collapse to a good approximation onto a single time-independent curve indicating dynamical scaling behavior. For experiment $B_{1}$ this collapse of the data holds to a lesser extent, since the scaled curves still increase somewhat with time. For experiment $\mathbf{B}_{2}$ this effect is even more pronounced and consequently no dynamical scaling is observed. We note, however, that for $\mathrm{B}_{2}$ curves have been used for the scaling analysis with transmission below $10 \%$.

Furukawa [53] has proposed the following scaling functions for off-critical and for critical quenches.

$$
\begin{aligned}
& \tilde{F}\left(q / q_{\max }\right)=\frac{3\left(q / q_{\max }\right)^{2}}{2+\left(q / q_{\max }\right)^{8}} \quad \text { (off critical), } \\
& \tilde{F}\left(q / q_{\max }\right)=\frac{4\left(q / q_{\max }\right)^{2}}{3+\left(q / q_{\max }\right)^{8}} \quad \text { (critical). }
\end{aligned}
$$

In Fig. 11 we have compared our scaled scattering intensities to the Furukawa scaling functions, which are scaled such that the peak height is the same as for the experimental curves. The scaled scattering curves resemble the shape predicted by Furukawa rather well. The scaled curves of experiment $A_{1}$ are similar to the offcritical Furukawa scaling function. The scattering curves of experiment $\mathrm{A}_{2}$ (slightly higher saturation) collapse onto a universal curve close to the critical Furukawa scaling function, apart from the rise in intensity at small wave vector. The scaled scattering curves for sample $\mathbf{B}_{1}$ are slightly broader than the Furukawa scaling functions. However, we have no plausible explanation for the detailed differences 

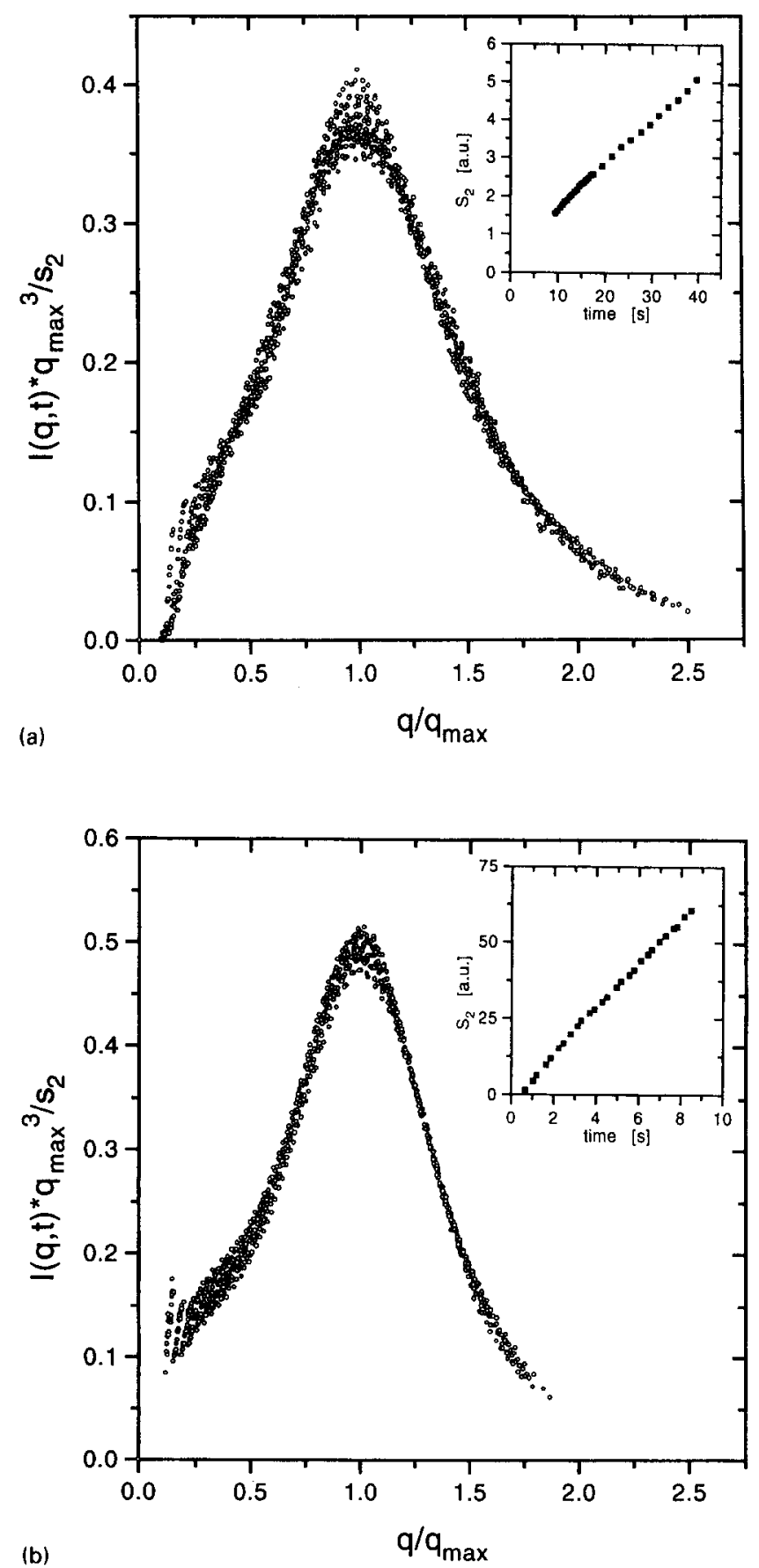

Fig. 10. Normalized scattering curves for (a) experiment $A_{1}$ (time interval: 9-40 s) and (b) experiment $A_{2}$ (time interval: 5-12 s) and for (c) experiment $B_{1}$ (time interval: $12.5-125 \mathrm{~s}$ ) and (d) experiment $B_{2}$ (time interval: $5.5-11 \mathrm{~s}$ ). The experimental data points are indicated by open circles. In the inset $\int I(q, t) q^{2} d q$ (denoted as $S_{2}$ ) is plotted versus time for the corresponding experiment. 

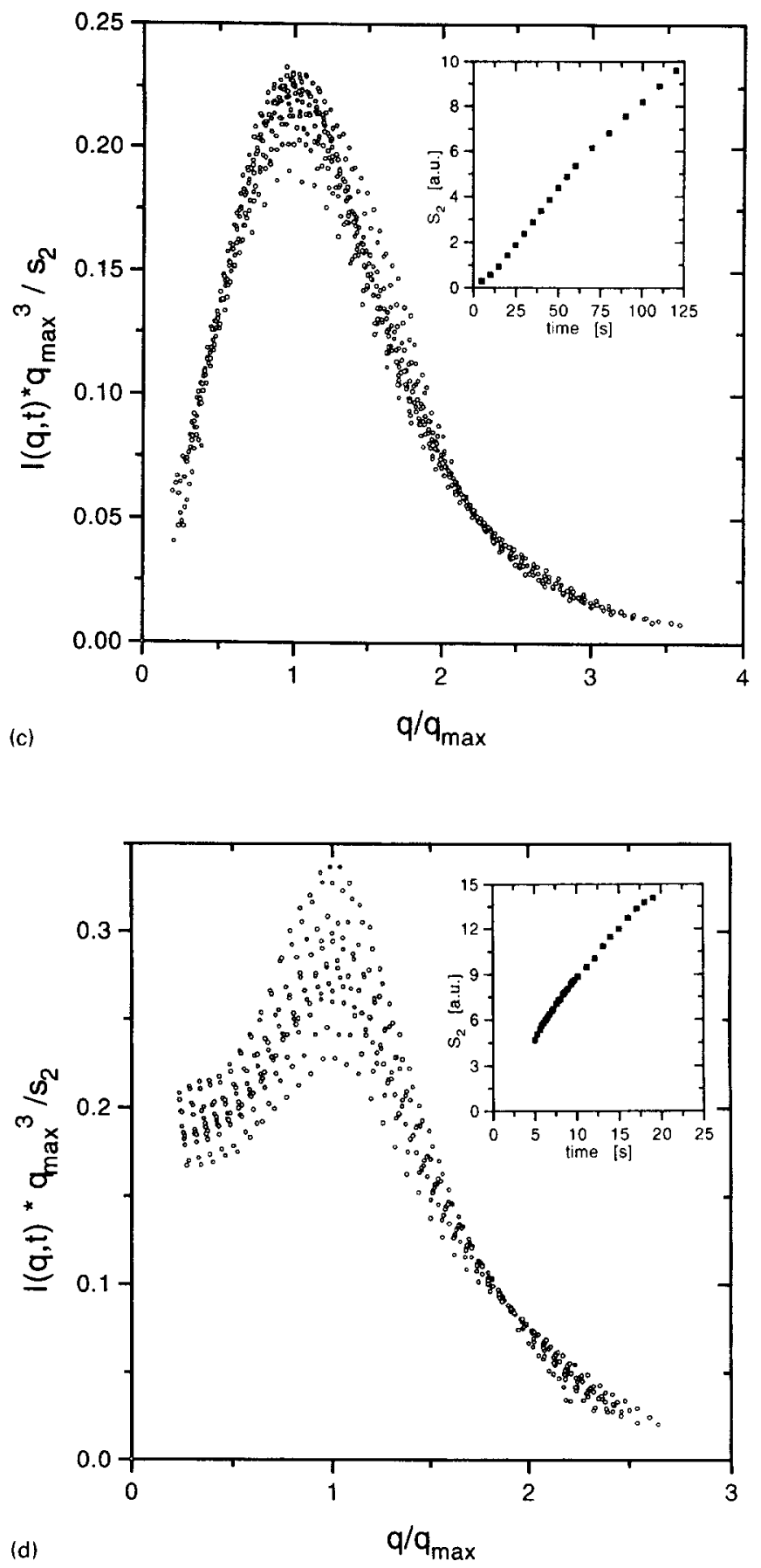

Fig. 10. Continued. 

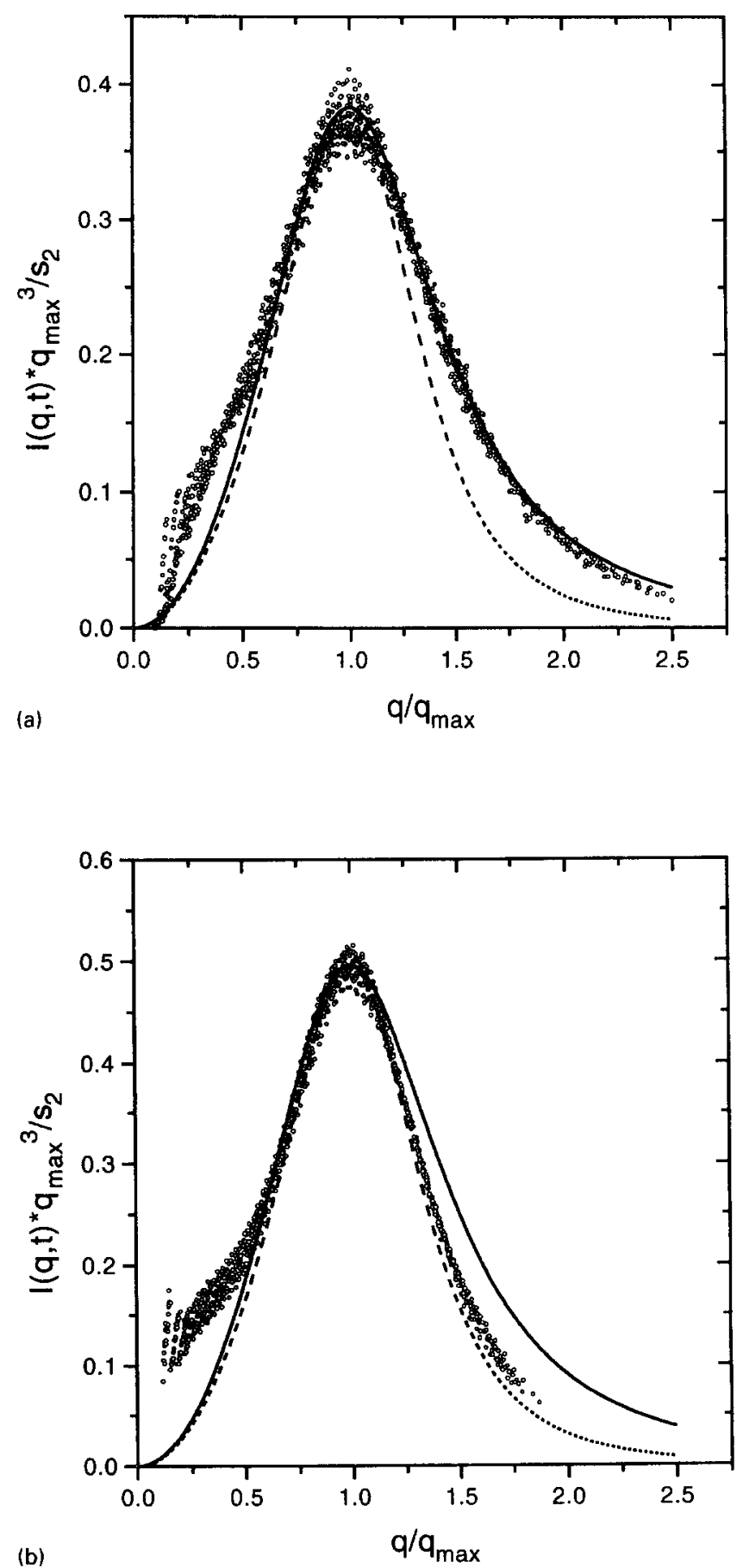

Fig. 11. Same normalized scattering functions as in Fig. 10 for experiment $A_{1}(a), A_{2}$ (b) and $B_{1}$ (c) Furukawa scaling functions (see text) have been included in all figures both for off-critical quenches (full line) and for critical quenches (dashed line). 


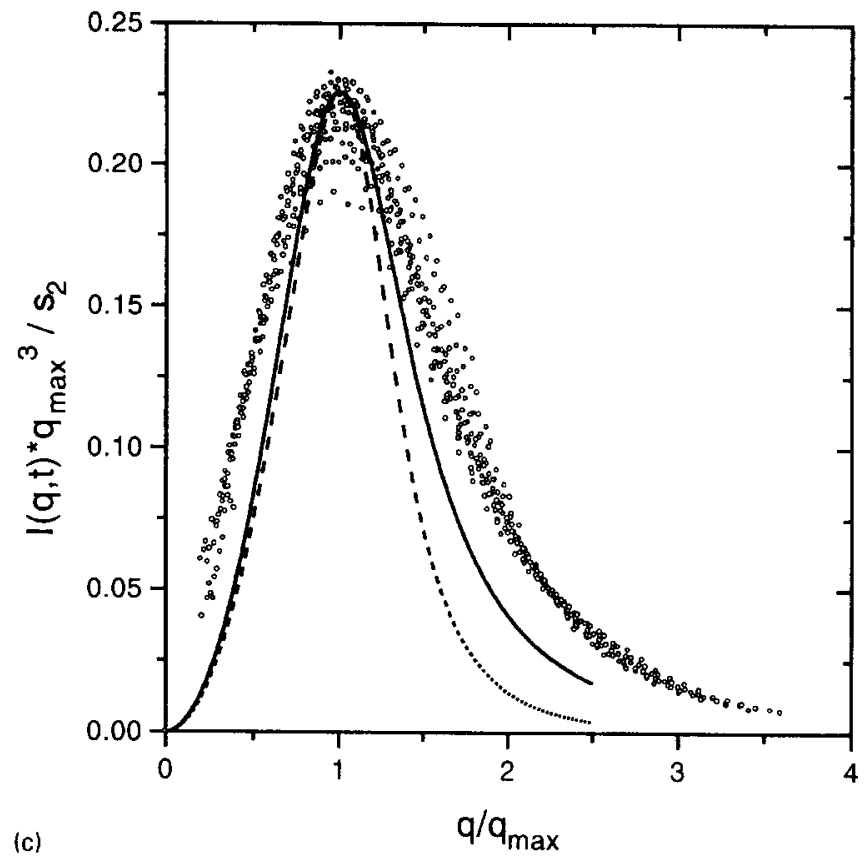

Fig. 11. Continued.

in peak widths. Nevertheless, it is remarkable that the unmixing processes in these colloid-polymer mixtures proceed similarly to the phase separating phenomena in binary liquid mixtures, polymer blends and metallic alloys. Different kinds of unmixing mixtures seem to satisfy a universal scaling behavior, indicating that the structure coarsening can be described with the time dependence of $q_{\max }$, which is correlated to the domain size.

\subsection{Light microscopy}

With microscopy significant differences in the evolution of morphology during phase separation have been observed for samples A, B and C. Sample A and B started as bicontinuous structures which coarsen in time. During the observation time ( $12 \mathrm{~min}$ ) sample A remained bicontinuous whereas in sample B after a few minutes small droplets were formed in the bulk. Sample C did not show a bicontinuous structure but small droplets were formed which grew larger than those observed in sample B. These droplets, presumably of polymer-rich minority phase, had a strong preference for the upper glass wall, where they coalesced forming a film. The difference in morphology should not necessarily be associated with the difference between the spinodal decomposition and the nucleation and growth mechanism. In the present case we only observed the droplets at late stages and an increase in supersaturation only gave rise to faster kinetics. 
In order to analyze the coarsening of the structure observed with light microscopy quantitatively, we performed 2D-Fourier transformations on digitized micrographs of experiment $\mathrm{A}_{1}$. Although these 2D-Fourier transforms do not represent the real space $3 \mathrm{D}$-system [54], they allowed us to probe the structure evolution on a longer time scale than accessible with small angle light scattering. Moreover, Guenoun et al. [54] found that the length scales determined with light scattering and with microscopy were similar.

The 2D-Fourier transforms showed a ring of high intensity, corresponding to a dominant length scale in the micrograph. For example, the typical length scale in experiment $A_{1} 14 \mathrm{~s}$ after homogenization was determined to be $7 \mu \mathrm{m}$. At the same time (Fig. 9(a)), light scattering produced a peak at $q_{\max }=1100 \mathrm{~mm}^{-1}$, corresponding to $l=2 \pi / q_{\max }=6 \mu \mathrm{m}$. Therefore, we assume that initially we study the same structures both with SALS and microscopy. However, the growth rate as observed with light microscopy seems to be significantly larger, since after $40 \mathrm{~s}$ we find a typical length scale of $9 \mu \mathrm{m}$ with SALS and of $17 \mu \mathrm{m}$ with microscopy. This difference in growth rate might either be due to the difference in geometry between the sample lying flat under the microscope and positioned upward for light scattering measurements, or might indicate that there is a small difference in concentration, such that the sample studied with microscopy phase separated at a slightly faster rate.

After about $150 \mathrm{~s}$ the micrographs of experiment $\mathrm{A}_{1}$ became blurred by hydrodynamic flow in the system. This results in a somewhat preferential orientation of domains in the unmixing mixture and a slight deformation of the Fourier transform pattern towards an ellipsoidal shape. With SALS, gravitational settling becomes important much earlier, after about $40 \mathrm{~s}$. Note however, that with SALS we studied the sample from the side and with the microscope from above.

\section{Conclusions}

As far as we know this is the first quantitative study of fluid-fluid phase separation kinetics in mixtures of colloid and non-adsorbing polymer. The unmixing process could be conveniently studied with small angle light scattering and light microscopy because of the appropriate characteristic length and time scales and the relative transparency of the mixtures. The time window of observation was limited by increasing turbidity that accompanied the unmixing process and the anisotropy induced by gravity when the length scales are larger than the capillary length. We obtained detailed information about the phase separation kinetics which could be analyzed within a framework which has been developed for simpler systems, such as binary liquid mixtures and polymer blends. Our data could not be described with linear Cahn-Hilliard theory. The coarsening of the typical length scale followed a $t^{1 / 3}$ behavior, which is indicative for growth by diffusion and coalescence of domains. For sufficiently low degrees of supersaturation the light scattering curves, 
normalized by $\int I(q, t) q^{2} d q$, satisfied dynamical scaling behavior. Close to the critical concentration, the scaling function resembles the forms predicted by Furukawa.

\section{Acknowledgements}

We would like to thank Bianca van der Zande and Gert Jan Vroege for preparing the stearyl coated ludox spheres and for discussions in an early stage of the experiments, John van Dijk (Leiden University) for performing the characterization of the polymers, Dr Stephen Picken (AKZO/NOBEL Central Research Laboratories Arnhem, The Netherlands) for his kindness to let us use his microscope and videocamera, and Maarten Terlou for analyzing the micrographs. We also would like to thank Professor A. Vrij for discussions about the interfacial tension. This work was supported by the Stichting voor Fundamenteel Onderzoek der Materie (Foundation for Fundamental Research on Matter) which is part of the Nederlandse Organisatie voor Wetenschappelijk Onderzoek (Netherlands Organization for the Advancement of Research). JVD acknowledges partial support through a Ramsay Memorial Fellowship.

\section{References}

[1] W.C.K. Poon, J.S. Selfe, M.B. Robertson, S.M. Ilett, A.D. Pirie and P.N. Pusey, J. Phys. II France 3 (1993) 1075.

[2] S.M. Ilett, A. Orrock, W.C.K. Poon and P.N. Pusey, Phys. Rev. E 51(2) (1995) 1344.

[3] F. Leal Calderon, J. Bibette and J. Bias, Europhys. Lett. 23(9) (1993) 653.

[4] P.N. Pusey, A.D. Pirie and W.C.K. Poon, Physica A 201 (1993) 322.

[5] W.C.K. Poon, A.D. Pirie and P.N. Pusey, submitted to Faraday Discussion.

[6] H. de Hek and A. Vrij, J. Coll. Interface Sci. 84(2) (1981) 409.

[7] H. de Hek and A. Vrij, J. Coll. Interface Sci. 88(1) (1982) 258.

[8] C. Smits, B. van der Most, J.K.G. Dhont and H.N.W. Lekkerkerker, Adv. Coll. Interface Sci. 42 (1992) 33.

[9] C. Pathmamanoharan, H. de Hek and A. Vrij, Colloid Polymer Sci. 259 (1981) 769.

[10] B. Vincent, J. Edwards, S. Emmett and R. Croot, Colloids Surfaces 31 (1988) 267.

[11] P.R. Sperry, J. Coll. Interface Sci. 99(1) (1984) 97.

[12] A.P. Gast, W.B. Russel and C.K. Hall, J. Coll. Interface Sci. 109(1) (1986) 161.

[13] P.D. Patel and W.B. Russel, J. Coll. Interface Sci. 131(1) (1989) 192.

[14] S. Asakura and F. Oosawa, J. Chem. Phys. 22 (1954) 1255.

[15] A. Vrij, Pure Appl. Chem. 48 (1976) 471.

[16] A.P. Gast, C.K. Hall and W.B. Russel, J. Coll. Interface Sci. 96 (1983) 251.

[17] M.M. Santore, W.B. Russel and R.K. Prud'homme, Macromolecules 22 (1989) 1317.

[18] H.N.W. Lekkerkerker, W.C.K. Poon, P.N. Pusey, A. Stroobants and P.B. Warren, Europhys. Lett 20(6) (1992) 559.

[19] E. Dickinson, R. Parker and M. Lal, Chem. Phys. Lett. 79 (1981) 578.

[20] E. Dickinson and R. Parker, J. Phys. France Lett. 46 (1985) L229.

[21] P.N. Pusey, J. Phys. 48 (1987) 709.

[22] J.S. Huang, W.I. Goldburg and A.W. Bjerkaas, Phys. Rev. Lett. 32(17) (1974) 921.

[23] Y.C. Chou and W.L. Goldburg, Phys. Rev. A 23(2) (1981) 858.

[24] J.E. Hilliard, in: Phase Transformations (American Society for Metals), ed. H.I. Aronson, Ohio (1970) p. 497. 
[25] J.W. Cahn, Transactions Metallurgical Society AIME 242 (1968) 166.

[26] F.S. Bates and P. Wiltzius, J. Chem. Phys. 91(5) (1989) 3258.

[27] R.H. Tromp, A.R. Rennie and R.A.L. Jones, Macromolecules 28 (1995) 4129.

[28] J.P. Wilcoxon, J.E. Martin and J. Odinek, J. Non-Cryst. Solids 172 (1994) 1142.

[29] P.W. Rouw, A.T.J.M. Woutersen, B.J. Ackerson and C.G. De Kruif, Physica A 156 (1989) 876.

[30] H. Verduin and J.K.G. Dhont, J. Coll. Interface Sci. 172 (1995) 425.

[31] J.W. Cahn and J.E. Hilliard, J. Chem. Phys. 28(2) (1958) 258.

[32] J.W. Cahn, J. Chem. Phys. 42 (1965) 93.

[33] J.W. Cahn and J.E. Hilliard, J. Chem. Phys 31(3) (1959) 688.

[34] J.W. Cahn, Acta Met. 9 (1961) 795.

[35] K. Binder and D.W. Heermann, in: Scaling Phenomena in Disordered Systems, eds. R. Pynn and A. Skjeltorp (Plenum, New York, 1985).

[36] K. Binder, in: Alloy Phase Stability, eds. G.M. Stocks and A. Gonis (Kluwer Academic Publishers, Dordrecht, 1989). p. 233.

[37] K. Binder, in: Phase Transformations of Materials (Materials Science and Technology, Vol. 5, Ch. 7) ed. P. Haasen (VCH Verlag, Weinheim, 1991) p. 405.

[38] K. Binder and D. Stauffer, Phys. Rev. Lett. 33 (1974) 1006.

[39] I.M. Lifshit and V.V. Slyozov, J. Phys. Chem. Solids 19 (1961) 35.

[40] C. Wagner, Z. Electrochem. 65 (1961) 581.

[41] E.D. Siggia, Phys. Rev. A 20(2) (1979) 595 .

[42] J. Marro, J.L. Lebowitz and M.H. Kalos, Phys. Rev. Lett. 43(4) (1979) 282.

[43] J.L. Lebowitz, J. Marro and M.H. Kalos, Act. Met. 30 (1982) 297.

[44] A.K. Van Helden and A. Vrij, J. Colloid Interface Sci. 76(2) (1980) 418.

[45] B. Chu, Laser Light Scattering (Academic Press, New York, 1969).

[46] K. Schätzel and B.J. Ackerson, Phys. Rev. E 48 (1993) 3766.

[47] T. Hashimoto, M. Itakura and H. Hasegawa, J. Chem. Phys. 85 (1986) 6118.

[48] K. Sasaki and T. Hashimoto, Macromolecules 17(12) (1984) 2818.

[49] K. Binder, H.L. Frisch and J. Jäckle, J. Chem. Phys. 85 (1986) 1505.

[50] V.I. Kalikmanov and G.G.J. Hofmans, J. Phys.: Condens. Matter 6 (1994) 2204.

[51] J.K.G. Dhont, An Introduction to Dynamics of Colloids, ch. 9 (Elsevier, Amsterdam, 1996).

[52] G. Porod, in: Small Angle X-ray Scattering, eds. O. Glatter and O. Kratky (Academic Press, London, 1982).

[53] H. Furukawa, Physica A 123 (1984) 497.

[54] P. Guenoun, R. Gastaud, F. Perrot and D. Beysens, Phys. Rev. A 36 (1987) 4876. 